\title{
Node Profiles of Symmetric Digital Search Trees: Concentration Properties
}

\author{
Michael Drmota \\ Institute for Discrete Mathematics and Geometry \\ Vienna University of Technology \\ 1040 Vienna \\ Austria \\ Hsien-Kuei Hwang ${ }^{\dagger}$ \\ Institute of Statistical Science \\ Academia Sinica \\ Taipei 115 \\ Taiwan
}

\author{
Michael Fuchs* \\ Department of Applied Mathematics \\ National Chiao Tung University \\ Hsinchu 300 \\ Taiwan
}

\author{
Ralph Neininger \\ Institute for Mathematics \\ Goethe University \\ 60054 Frankfurt a.M. \\ Germany
}

September 30, 2020

\begin{abstract}
We give a detailed asymptotic analysis of the profiles of random symmetric digital search trees, which are in close connection with the performance of the search complexity of random queries in such trees. While the expected profiles have been analyzed for several decades, the analysis of the variance turns out to be very difficult and challenging, and requires the combination of several different analytic techniques, including Mellin and Laplace transforms, analytic de-Poissonization, and Laplace convolutions. Our results imply concentration of the profiles in the range where the mean tends to infinity. Moreover, we also obtain a two-point concentration for the distributions of the height and the saturation level.
\end{abstract}

AMS 2010 subject classifications. Primary 05A16,60C05, 68Q25; secondary 68P05, 60F05.

Key words. Digital search tree, level profile, two-point concentration, double-indexed recurrence, asymptotic transfer, Poissonization, Laplace transform, Mellin transform.

\section{Introduction and Results}

Digital trees are fundamental data structures for words or alphabets in computer algorithms whose analysis has attracted much attention over the last half century. One major such variant is the digital search tree (DST for short), introduced by Coffman and Eve in 1970 [5] (see also [22] for more information). Such structures are closely related to the popular Lempel-Ziv compression scheme, and their asymptotic behaviors under random inputs are often more challenging than those for other digital tree families because of the natural occurrence of differential-functional equations instead of purely algebraic-functional equations.

\footnotetext{
${ }^{*}$ Partially supported by MOST under the grants MOST-104-2923-M-009-006-MY3 and MOST-109-2115-M-004-003MY2.

${ }^{\dagger}$ Partially supported by an Investigator Award from Academia Sinica under the Grant AS-IA-104-M03.
} 
We begin with the definition of DST, which is the main object of study in this paper. In the simplest situation, it is built from digital data consisting of a sequence of records in the form of 0-1 strings. The first record is stored at the root of the tree. All other records are distributed to the left- or right-subtree according as their first bit being 0 or 1 , respectively, and retain their relative order. The subtrees of the root are then built according to the same rules but by using the $j$ th digit at level $j$ in further directing the strings to their respective subtrees. The splitting process stops when the size of the subtree is either zero or one. Note that the resulting tree is a binary tree with internal nodes holding the records. External nodes, which represent places where potential records can be inserted, are often added to the tree (in fact, two external nodes are automatically created in the algorithmic implementation for each new internal node); see Figure 1 for an example of a DST built from five records (internal nodes are represented by rectangles and external nodes by circles).

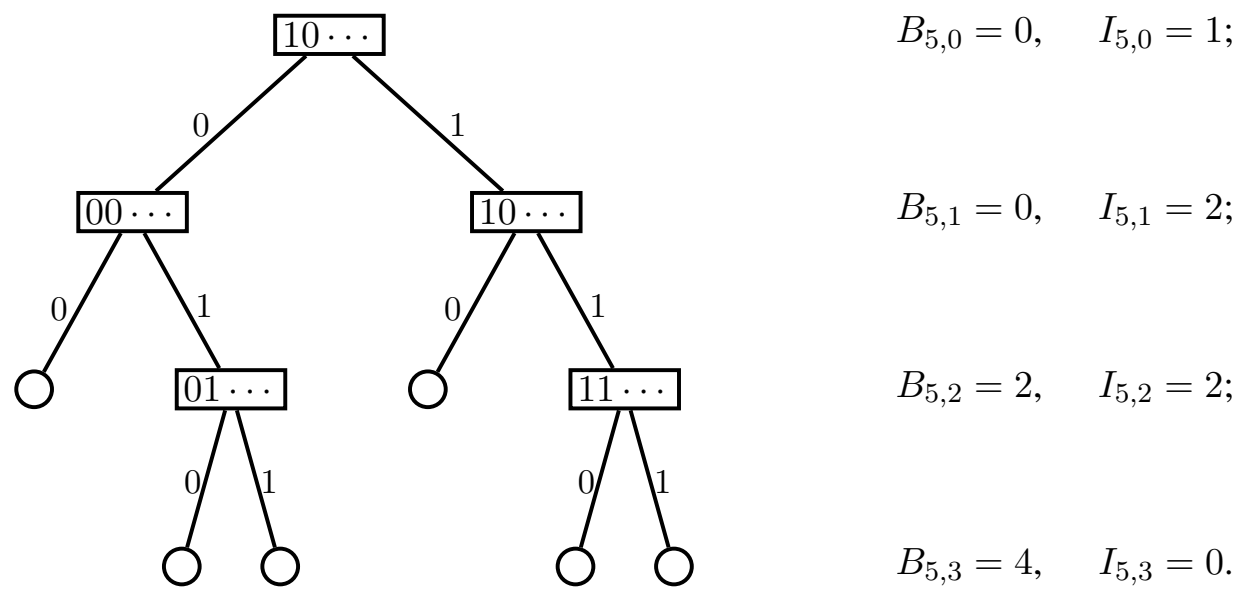

Figure 1: A DST built from 5 records with its profiles.

For the purpose of analysis, we assume that bits in the input strings are independent and identically distributed with a common $\operatorname{Bernoulli}(p)$ random variable with $0<p<1$. Throughout this paper, we fix $p=\frac{1}{2}$, namely, we consider only the symmetric case. This random model is called the symmetric Bernoulli model and the corresponding random tree is referred to as a random symmetric DST. It represents a simple model with reasonably good predictive power in general (for example, results holding in the Bernoulli model often have similar forms in more general Markov models; see [25]).

Under such a random model, we study in this paper the external and internal node profiles (referred to as the profiles for short) which are defined as follows: the external profile of a random symmetric DST of size $n$ is a double-indexed sequence of random variables $B_{n, k}$ which counts the number of external nodes at (horizontal) level $k$; the internal profile $I_{n, k}$ is similarly defined (with external nodes replaced by internal nodes).

Profiles are fine shape characteristics encoding the level silhouette of the tree and they are closely connected to many other shape parameters such as height, width, total path length, saturation or fill-up level, and successful and unsuccessful search. In particular, we will discuss the unsuccessful search (or the depth), the height and the saturation level:

- Unsuccessful search $U_{n}$ : the distance from the root to a randomly chosen external node with its distribution given by

$$
\mathbb{P}\left(U_{n}=k\right)=\frac{\mathbb{E}\left(B_{n, k}\right)}{n+1} .
$$

- Height: the length of the longest path from the root to an external node, or $\max \left\{k: B_{n, k}>0\right\}$; 
- Saturation level: the last level from the root which is completely filled with internal nodes, or $\max \left\{k: I_{n, k}=2^{k}\right\}$.

See for example $[8,15]$ and the references therein for more shape parameters in DSTs.

Historically, the external profile was among the very first shape parameters analyzed on DSTs due to the connection (1) to the unsuccessful search; see Knuth [22] and Konheim and Newman [23]. Yet the understanding of the profiles of symmetric DSTs has remained incomplete. Table 1 summarizes the current status for the profiles of tries, Patricia tries and DSTs, the latter two representing other major classes of digital trees.

\begin{tabular}{ccccc} 
Tree types & $p=q ?$ & Mean & Variance & CLT \\
\hline \multirow{2}{*}{ Tries } & $0<p<1$ & {$[30]$} & {$[30]$} & {$[30]$} \\
\cline { 2 - 5 } Patricia Tries & $p=\frac{1}{2}$ & {$[27]$} & $?$ & $?$ \\
& $p \neq \frac{1}{2}$ & {$[10,27,26]$} & {$[10,26]$} & {$[26]$} \\
\cline { 2 - 5 } DSTs & $p=\frac{1}{2}$ & {$[24,9]$} & this paper & next paper \\
& $p \neq \frac{1}{2}$ & {$[9]$} & {$[18]$} & $?$ \\
\hline
\end{tabular}

Table 1: A summary of the analysis in distribution of profiles in the three major classes of digital trees under the Bernoulli model.

Briefly, in the case of random tries, the mean, the variance and the asymptotic normality of both profiles under the symmetric and asymmetric models are fully clarified in [30]. For Patricia tries, the expected profiles were studied in [27] for both symmetric and asymmetric models. Then the asymptotic variance and the asymptotic normality of the profiles, inter alia, under the asymmetric model are established in the recent papers $[10,26]$.

As regards symmetric DSTs, Louchard [24], following [22, 23], derived an explicit expression for the expected profiles; see also [8, 9, 28, 31]. Louchard also obtained an asymptotic approximation for the mean profiles in the most important range $k=\log _{2} n+\mathcal{O}(1)$ (where most nodes lie), characterizing the asymptotic distribution of unsuccessful and successful search. These results were later extended in $[8,9,21,28]$. We broaden the study in this paper to the variance of the profiles for which an arduous analysis is carried out. From our results we obtain concentration of the profiles around the expected profile in the range where the mean becomes unbounded. This suggests that an asymptotic normality result (in the sense of convergence in distribution) holds in this range, too. We will deal with this question in a companion paper due to very different techniques required. Moreover, we will apply our results to the height and the saturation level. See also [6, 17, 25, 32] for other parameters and different types of results on profiles in DSTs.

We now state our results, focusing on the external profile. The corresponding results for the internal profile will be given in Section 4. First, we introduce the following function and sequence that are ubiquitous in the analysis of DSTs; see [22].

$$
Q(z)=\prod_{\ell \geqslant 1}\left(1-2^{-\ell} z\right) \quad \text { and } \quad Q_{n}=\prod_{1 \leqslant \ell \leqslant n}\left(1-2^{-\ell}\right)=\frac{Q(1)}{Q\left(2^{-n}\right)} .
$$

Note that $\lim _{n \rightarrow \infty} Q_{n}$ exists and equals $Q(1)=: Q_{\infty}$.

In the next section, we will derive the following (largely known) result (see [8]) for the mean of the external profile.

Theorem 1. The mean of the external profile satisfies

$$
\mathbb{E}\left(B_{n, k}\right)=2^{k} F\left(2^{-k} n\right)+\mathcal{O}(1),
$$


uniformly for $0 \leqslant k \leqslant n$, where $F(x)$ is a positive function on $\mathbb{R}^{+}$defined by

$$
F(x)=\sum_{j \geqslant 0} \frac{(-1)^{j} 2^{-\left(\begin{array}{c}
j \\
2
\end{array}\right)}}{Q_{j} Q_{\infty}} e^{-2^{j} x} .
$$

The proof of (2) for small $k$, or more precisely, for $k$ such that $2^{-k} n \rightarrow \infty$, will follow readily by simple elementary arguments, whereas that for the remaining range will rely on complex-analytic tools. More precisely, when $2^{-k} n \rightarrow \infty$ and $k \geqslant 1$, we will show that

$$
\mathbb{E}\left(B_{n, k}\right)=\frac{2^{k}}{Q_{k}}\left(1-2^{-k}\right)^{n}\left(1+\mathcal{O}\left(e^{-\frac{n}{2^{k}-1}}\right)\right),
$$

which is stronger than (2) if $2^{k} F\left(2^{-k} n\right)=\mathcal{O}(1)$.

Remark 1. Note that (4) indeed holds for all $n$ and $k$ but is more useful in the range when $2^{-k} n \rightarrow \infty$.
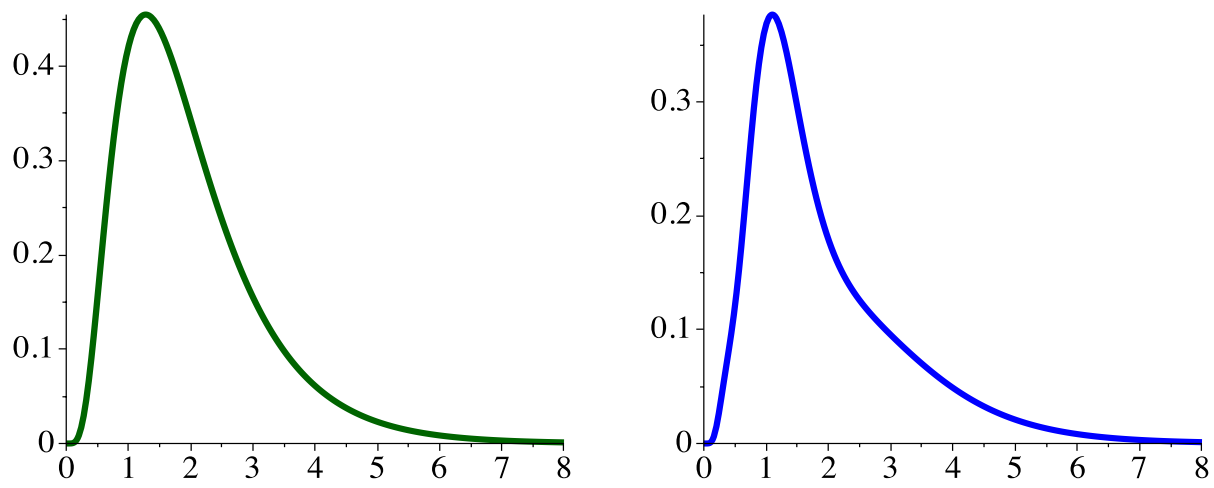

Figure 2: The functions $F$ (left) and $G$ (right).

On the other hand, the relation (2) is only a (useful) asymptotic approximation if the first term on the right-hand side is not bounded for large $n$. Thus, to understand when this holds, we derive more precise asymptotic approximations of $F(x)$ for large and small $x$; see Figure 2 (left) for a graphical rendering of $F$.

Observe first that the series definition (3) of $F$ extends to complex parameter $z$ with $\Re(z) \geqslant 0$ and is itself an asymptotic expansion for large $|z|$ :

$$
F(z)=\frac{e^{-z}}{Q_{\infty}}+\mathcal{O}\left(e^{-2 \Re(z)}\right), \quad(\Re(z) \geqslant 0) .
$$

On the other hand, for small $x$ with $X:=\frac{1}{x \log 2}$ (see Proposition 1),

$$
F(x)=\sqrt{\frac{\log 2}{2 \pi}}(1+o(1)) X^{\frac{1}{2}+\frac{1}{\log 2}} \exp \left(-\frac{(\log (X \log X))^{2}}{2 \log 2}-P\left(\log _{2}(X \log X)\right)\right),
$$

with $P(t), t \in \mathbb{R}$, a 1-periodic function whose Fourier series is given explicitly by

$$
P(t):=\frac{\log 2}{12}+\frac{\pi^{2}}{6 \log 2}-\sum_{j \geqslant 1} \frac{\cos (2 j \pi t)}{j \sinh \left(\frac{2 j \pi^{2}}{\log 2}\right)} .
$$

Note that the series in (7), representing the fluctuating part of $P(t), t \in \mathbb{R}$, has a (peak-to-peak) amplitude less than $1.8 \times 10^{-12}$. Both expansions (6) and (7) can be extended to complex parameter; see Proposition 1.

While it is well anticipated (from known results for tries and Patricia tries) that $\operatorname{Var}\left(B_{n, k}\right)$ is asymptotically of the same form as (2) for $\mathbb{E}\left(B_{n, k}\right)$ in most ranges of $k$ of interest, the function involved is surprisingly very complicated, as shown in (9) below; see also Figure 2 (right). 
Theorem 2. The variance of the external profile satisfies

$$
\operatorname{Var}\left(B_{n, k}\right)=2^{k} G\left(2^{-k} n\right)+\mathcal{O}(1),
$$

uniformly for $0 \leqslant k \leqslant n$, where $G(x)$ is a positive function on $\mathbb{R}^{+}$defined by

$$
G(x)=\sum_{j, r \geqslant 0} \sum_{0 \leqslant h, \ell \leqslant j} \frac{(-1)^{r+h+\ell} 2^{-j-\left(\begin{array}{c}
r \\
2
\end{array}\right)-\left(\begin{array}{c}
h \\
2
\end{array}\right)-\left(\begin{array}{c}
\ell \\
2
\end{array}\right)+2 h+2 \ell}}{Q_{\infty} Q_{r} Q_{h} Q_{j-h} Q_{\ell} Q_{j-\ell}} \varphi\left(2^{j+r}, 2^{h}+2^{\ell} ; x\right),
$$

with

$$
\varphi(u, v ; x)=e^{-v x} \int_{0}^{x}(x-t) e^{-(u-v) t} \mathrm{~d} t= \begin{cases}e^{-v x} \frac{e^{-(u-v) x}-1+(u-v) x}{(u-v)^{2}}, & \text { if } u \neq v \\ \frac{1}{2} x^{2} e^{-u x}, & \text { if } u=v .\end{cases}
$$

Remark 2. In the case when $2^{-k} n \rightarrow \infty$, we will in fact prove that

$$
\mathbb{E}\left(B_{n, k}\right) \sim \operatorname{Var}\left(B_{n, k}\right) .
$$

Despite of its complicated form, the function $G$ is very close to $F$ in the following sense (see Section 3):

$$
G(x) \sim \begin{cases}F(x), & \text { if } x \rightarrow \infty \\ 2 F(x), & \text { if } x \rightarrow 0\end{cases}
$$

see also [30] for the same type of results for symmetric tries, and Devroye [7] for a general bound for the profile variance. If $x \rightarrow \infty$, then a more precise approximation is

$$
G(x)=\frac{e^{-x}}{Q_{\infty}}+\mathcal{O}\left(x e^{-2 x}\right),
$$

where the second-order term differs from that of $F$; see (5).

The two theorems imply that the mean and the variance have very similar behaviors. In particular, they tend to infinity in the same range of $k$.

Corollary 1. For large $n$ and $0 \leqslant k \leqslant n, \mathbb{E}\left(B_{n, k}\right) \rightarrow \infty$ if and only if $\operatorname{Var}\left(B_{n, k}\right) \rightarrow \infty$. In fact, $\mathbb{E}\left(B_{n, k}\right)$ and $\operatorname{Var}\left(B_{n, k}\right)$ are of the same order of magnitude in this range.

We now describe the range where the mean and the variance tend to infinity. Define two functions of $n$ :

$$
\begin{aligned}
& k_{s}:=\log _{2} n-\log _{2} \log n+\frac{\log _{2} \log n}{\log n}, \\
& k_{h}:=\log _{2} n+\sqrt{2 \log _{2} n}-\frac{1}{2} \log _{2} \log _{2} n+1+\frac{1}{\log 2}-\frac{3 \log \log n}{4 \sqrt{2(\log n)(\log 2)}} .
\end{aligned}
$$

Corollary 2. The mean and the variance of $B_{n, k}$ tend to infinity if and only if there exists a positive sequence $\omega_{n}$ tending to infinity with $n$ such that

$$
k_{s}+\frac{\omega_{n}}{\log n} \leqslant k \leqslant k_{h}-\frac{\omega_{n}}{\sqrt{\log n}} .
$$

The proof of this follows from a straightforward computation and is left to the reader; see Section 5 for similar computations. Note that the range is very small (or almost all nodes are concentrated at these levels). For convenience, we refer to (14) as the central range.

Since $\mathbb{E}\left(B_{n, k}\right)$ and $\operatorname{Var}\left(B_{n, k}\right)$ are of the same order of magnitude as $\operatorname{Var}\left(B_{n, k}\right) \rightarrow \infty$, we immediately get the following property. 
Corollary 3. If $\operatorname{Var}\left(B_{n, k}\right) \rightarrow \infty$, then the external profile is asymptotically concentrated around its expected value:

$$
\frac{B_{n, k}}{\mathbb{E}\left(B_{n, k}\right)} \stackrel{P}{\longrightarrow} 1
$$

where $\stackrel{P}{\longrightarrow}$ denotes convergence in probability.

Corollary 3 suggests that the profile follows the central limit theorem in the central range:

$$
\frac{B_{n, k}-\mathbb{E}\left(B_{n, k}\right)}{\sqrt{\operatorname{Var}\left(B_{n, k}\right)}} \stackrel{d}{\longrightarrow} \mathscr{N}(0,1),
$$

where $\mathscr{N}(0,1)$ denotes the standard normal distribution. Indeed, we can prove (15) when the variance tends to infinity not too slowly by using the methods of this paper and the contraction method; compare with Neininger and Rüschendorf [29]. However, in order to obtain (15) for the whole central range, a finer analysis of the asymptotic behaviors of $\mathbb{E}\left(B_{n, k}\right)$ and $\operatorname{Var}\left(B_{n, k}\right)$ is required, as well as a delicate bivariate asymptotic transfer result for recurrences of the type (19). These will be addressed in a companion paper.

Results of a very similar nature for the internal profile are given in Section 4.

These new results for the internal and external profile have many consequences in view of their close connections to other shape parameters. We content ourselves here with stating an application to the height $H_{n}$ of DSTs, which is related to $B_{n, k}$ by $H_{n}:=\max \left\{k: B_{n, k}>0\right\}$; see Section 5 for other consequences.

Theorem 3. Define $k_{H}$ as

$$
k_{H}=\left\lfloor\log _{2} n+\sqrt{2 \log _{2} n}-\frac{1}{2} \log _{2} \log _{2} n+\frac{1}{\log 2}\right\rfloor,
$$

which is at the upper boundary of the central range (14). Then the distribution of $H_{n}$ is concentrated at the two points $k_{H}$ and $k_{H}+1$ :

$$
\mathbb{P}\left(H_{n}=k_{H} \text { or } H_{n}=k_{H}+1\right) \rightarrow 1, \quad(n \rightarrow \infty) .
$$

The possibility that such a result might hold was mentioned in [1] for a closely related model; a heuristic derivation was given in [19]. See also [2, 3] for other two-point approximation results in probability theory.

It is interesting to compare (17) with known results for the height of tries and those for Patricia tries, which we summarize in Table 2; see Flajolet [11] for the height of symmetric tries, and Knessl and Szpankowski [20] for that of Patricia tries (with only non-rigorous proofs).

\begin{tabular}{cccc} 
Tree types & Expected height & $\begin{array}{c}\text { Discrete } \\
\text { concentration }\end{array}$ & References \\
\hline Tries & $2 \log _{2} n+\mathcal{O}(1)$ & no & {$[11]$} \\
Patricia tries & $\log _{2} n+\sqrt{2 \log _{2} n}+\mathcal{O}(1)$ & at 3 pts & [20] (non-rigorous) \\
& $\log _{2} n+\sqrt{2 \log _{2} n}$ & at 2 pts & this paper \\
DSTs & $-\frac{1}{2} \log _{2} \log _{2} n+\mathcal{O}(1)$ & & \\
\hline
\end{tabular}

Table 2: A comparison of the height of random symmetric tries, Patricia tries and DSTs. 
We describe briefly the methods and tools used in proving Theorems $1-2$, which both start with the following distributional recurrence

$$
B_{n, k} \stackrel{d}{=} B_{J_{n}, k-1}+B_{n-1-J_{n}, k-1}^{*}, \quad(n, k \geqslant 1),
$$

with the boundary conditions $B_{0,0}=1, B_{0, k}=0$ for $k \geqslant 1, B_{n, 0}=0$ for $n \geqslant 1$, where $J_{n}=$ $\operatorname{Binomial}\left(n-1, \frac{1}{2}\right)$, and $B_{n, k}^{*}$ is an independent copy of $B_{n, k}$.

To derive the asymptotic approximations of the mean (Theorem 1) and the variance (Theorem 2), we rely on the property, in view of (18), that all moments of $B_{n, k}$ satisfy recurrences of the following type:

$$
a_{n, k}=2^{2-n} \sum_{0 \leqslant j<n}\left(\begin{array}{c}
n-1 \\
j
\end{array}\right) a_{j, k-1}+b_{n, k}
$$

for some given sequence $b_{n, k}$. This recurrence looks standard but the complication here comes from the dependence of $k$ on $n$. When $k$ is small, more precisely, when $2^{-k} n \rightarrow \infty$, the tree shape at these levels has little variation and thus both mean and variance can be treated by simple elementary arguments. The hard ranges are when $2^{-k} n \asymp 1$ and $2^{-k} n \rightarrow 0$ for which our arguments are built upon the idea of Poissonization by defining the Poisson generating functions

$$
\tilde{A}_{k}(z)=e^{-z} \sum_{n \geqslant 0} a_{n, k} \frac{z^{n}}{n !} \quad \text { and } \quad \tilde{B}_{k}(z)=e^{-z} \sum_{n \geqslant 0} b_{n, k} \frac{z^{n}}{n !} .
$$

Then (19) is translated into the differential-functional equation

$$
\tilde{A}_{k}(z)+\tilde{A}_{k}^{\prime}(z)=2 \tilde{A}_{k-1}\left(\frac{1}{2} z\right)+\tilde{B}_{k}(z),
$$

which amounts to describing the moments in the Poisson model. This equation is solved via Laplace transform techniques, which lead to exact and asymptotic expressions whose asymptotic properties are further examined via Mellin transform, saddle-point method and again Laplace transform. Finally, we translate the results in the Poisson model to those in the Bernoulli model via de-Poissonization.

While these procedures are by now standard (see $[14,15]$ ) and work well for the mean, the analysis of the variance is more subtle. Here, the most crucial step is to introduce a Poissonized variance in the Poisson model (see again $[14,15]$ ) so as to provide an asymptotic equivalent to the variance after de-Poissonization. An appropriate adaptation in the current situation is to define the function

$$
\tilde{V}_{k}(z):=\tilde{M}_{k, 2}(z)-\tilde{M}_{k, 1}(z)^{2}-z \tilde{M}_{k, 1}^{\prime}(z)^{2},
$$

where $\tilde{M}_{k, 2}(z)$ and $\tilde{M}_{k, 1}(z)$ denote the Poisson generating functions of the second moment and the first moment of $B_{n, k}$, respectively. Then we show that in the central range $\tilde{V}_{k}(z)$ is well-approximated by $2^{k} G\left(2^{-k} z\right)$ for large $|z|$, and that $\tilde{V}_{k}(n)$ is asymptotically equivalent to $\operatorname{Var}\left(B_{n, k}\right)$. Once these are clarified, the next challenge is the asymptotic behavior of $G(z)$, notably for small $|z|$, which turns out to be the most technical part of this paper (see Proposition 3), largely due to the complicated form of the Laplace transform of $G(z)$ (see (46) and (47)) and the uniformity required for large parameters (see Lemma 8).

Finally, Theorem 3 and some related properties will be proved in Section 5 by the first and second moment method, relying on the estimates from Sections 2-4. The corresponding asymptotic properties for the internal profiles will be given in Section 4.

An extended abstract of this paper (entitled External Profile of Symmetric Digital Search Trees) by the same authors has appeared in the Proceedings of the Fourteenth Workshop on Analytic Algorithmics and Combinatorics (ANALCO17), and contains Theorems 1-3 and sketches of the proofs of the first two. With the exception of the limiting distributions, the current paper provides the proofs and derives additionally the same types of asymptotic approximations for the internal profile and discusses some of their consequences. 


\section{Expected Values of the External Profile}

In this section, we prove Theorem 1 for $\mathbb{E}\left(B_{n, k}\right)$. As mentioned in the Introduction, most results given here are known. Nevertheless, we provide detailed proofs because the analysis of the variance will follow the same pattern.

We start from (18). Write $\mu_{n, k}=\mathbb{E}\left(B_{n, k}\right)$. Then

$$
\mu_{n, k}=2^{2-n} \sum_{0 \leqslant j<n}\left(\begin{array}{c}
n-1 \\
j
\end{array}\right) \mu_{j, k-1}, \quad(n, k \geqslant 1),
$$

with the boundary conditions $\mu_{0,0}=1$, and $\mu_{n, 0}=\mu_{0, k}=0$ for $n, k \geqslant 1$. We then consider the Poisson generating function

$$
\tilde{M}_{k, 1}(z):=e^{-z} \sum_{n \geqslant 0} \mu_{n, k} \frac{z^{n}}{n !}, \quad(k \geqslant 0),
$$

which satisfies the differential-functional equation

$$
\tilde{M}_{k, 1}(z)+\tilde{M}_{k, 1}^{\prime}(z)=2 \tilde{M}_{k-1,1}\left(\frac{1}{2} z\right), \quad(k \geqslant 1),
$$

with $\tilde{M}_{0,1}(z)=e^{-z}$.

We now solve this differential-functional equation using Laplace transform, which, by inverting and taking coefficients, leads to an exact expression for $\mu_{n, k}$.

\subsection{Exact Expressions}

To solve (20), we apply the Laplace transform (subsequently denoted by $\mathscr{L}[\cdot ; s]$ ) on both sides of (20), and obtain

$$
\mathscr{L}\left[\tilde{M}_{k, 1}(z) ; s\right]=\frac{4}{s+1} \mathscr{L}\left[\tilde{M}_{k-1,1}(z) ; 2 s\right], \quad(k \geqslant 1),
$$

with $\mathscr{L}\left[\tilde{M}_{0,1}(z) ; s\right]=\frac{1}{s+1}$. A direct iteration then yields

$$
\mathscr{L}\left[\tilde{M}_{k, 1}(z) ; s\right]=\frac{4^{k}}{(s+1)(2 s+1) \cdots\left(2^{k} s+1\right)},
$$

for $k \geqslant 0$. By partial fraction expansion, we see that

$$
\mathscr{L}\left[\tilde{M}_{k, 1}(z) ; s\right]=2^{k} \sum_{0 \leqslant j \leqslant k} \frac{(-1)^{j} 2^{-\left(\begin{array}{c}
j \\
2
\end{array}\right)}}{Q_{j} Q_{k-j}} \cdot \frac{1}{s+2^{j-k}},
$$

which, by term-by-term inversion, gives

$$
\tilde{M}_{k, 1}(z)=2^{k} \sum_{0 \leqslant j \leqslant k} \frac{(-1)^{j} 2^{-\left(\begin{array}{l}
j \\
2
\end{array}\right)}}{Q_{j} Q_{k-j}} e^{-2^{j-k} z}, \quad(k \geqslant 0) .
$$

From this, we obtain the closed-form expression for the expected profile (first derived in [24])

$$
\mu_{n, k}=2^{k} \sum_{0 \leqslant j \leqslant k} \frac{(-1)^{j} 2^{-\left(\begin{array}{c}
j \\
2
\end{array}\right)}}{Q_{j} Q_{k-j}}\left(1-2^{j-k}\right)^{n} .
$$

We now examine the asymptotic aspects. 


\subsection{Asymptotics of $\mu_{n, k}$}

If $2^{-k} n \rightarrow \infty$, then an expansion for the mean can be derived by elementary arguments because the term in (24) with $j=0$ is dominant. More precisely, we have

$$
\mu_{n, k}=\frac{2^{k}}{Q_{k}}\left(1-2^{-k}\right)^{n}\left(1+\mathcal{O}\left(\frac{\left(1-2^{1-k}\right)^{n}}{\left(1-2^{-k}\right)^{n}}\right)\right)
$$

where the error term is bounded above by

$$
\frac{\left(1-2^{1-k}\right)^{n}}{\left(1-2^{-k}\right)^{n}}=\exp \left(-n \sum_{\ell \geqslant 1} \frac{2^{\ell}-1}{\ell 2^{k \ell}}\right) \leqslant \exp \left(-\frac{n}{2^{k}-1}\right) \quad(k \geqslant 1) .
$$

Substituting this into (25) proves the asymptotic estimate (4) for $\mu_{n, k}$ when $2^{-k} n \rightarrow \infty$ and $k \geqslant 0$.

If $2^{-k} n=\mathcal{O}(1)$, then no single term in (24) is dominating and $2^{-r k} n \rightarrow 0$ for $r \geqslant 2$, so we readily obtain, again by (24) (approximating $(1-x)^{n}$ by $e^{-x n}$ and by extending $k$ to infinity), $\mu_{n, k} \sim 2^{k} F\left(2^{-k} n\right)$, but the asymptotics of $F$ for small parameter remains unclear. We use instead the Poissonization techniques (see $[15,16]$ ) to derive the required asymptotic approximation of $\mu_{n, k}$; see Theorem 1.

We derive first a simple bound for $F(z)$ and its derivatives.

Lemma 1. For $m \geqslant 0$ and $\Re(z) \geqslant 0$, the mth derivative of $F$ satisfies the uniform bound

$$
\sup _{\Re(z) \geqslant 0}\left|F^{(m)}(z)\right|=\mathcal{O}\left(2^{\left(\begin{array}{c}
m+1 \\
2
\end{array}\right)}\right),
$$

where the implied constant is absolute.

Proof. By the definition (3):

$$
\left|F^{(m)}(z)\right| \leqslant \sum_{j \geqslant 0} \frac{2^{-\left(\begin{array}{c}
j \\
2
\end{array}\right)+j m}}{Q_{j} Q_{\infty}} e^{-2^{j} \Re(z)} \leqslant Q_{\infty}^{-2} e^{-\Re(z)} \sum_{j \geqslant 0} 2^{-\left(\begin{array}{c}
j \\
2
\end{array}\right)+j m} .
$$

Now

$$
\sum_{j \geqslant 0} 2^{-\left(\begin{array}{l}
j \\
2
\end{array}\right)+j m} \leqslant 2^{\frac{1}{2}\left(m+\frac{1}{2}\right)^{2}} \sum_{j \in \mathbb{Z}} 2^{-\frac{1}{2}\left(j-m-\frac{1}{2}\right)^{2}}=2^{\frac{1}{2}\left(m+\frac{1}{2}\right)^{2}+1} \sum_{j \geqslant 1} 2^{-\frac{1}{2}\left(j-\frac{1}{2}\right)^{2}} .
$$

This proves the uniform bound (27).

We then show that (23) can be brought into the following more useful form (both exact and asymptotic).

Lemma 2. For $\Re(z) \geqslant 0$ and $k \geqslant 1$, the Poisson generating function $\tilde{M}_{k, 1}(z)$ of the expected profile $\mu_{n, k}$ satisfies

$$
\tilde{M}_{k, 1}(z)=2^{k} \sum_{m \geqslant 0} \frac{2^{-\left(\begin{array}{c}
m+1 \\
2
\end{array}\right)-k m}}{Q_{m}} F^{(m)}\left(2^{-k} z\right),
$$

where $F(z)$ is given in (3).

Proof. By Euler's identity (see [4, Corollary 2.2])

$$
\sum_{j \geqslant 0} \frac{(-1)^{j} q^{\left(\begin{array}{l}
j \\
2
\end{array}\right)}}{(1-q)\left(1-q^{2}\right) \cdots\left(1-q^{j}\right)} z^{j}=\prod_{\ell \geqslant 0}\left(1-q^{\ell} z\right), \quad(0<q<1),
$$


we have

$$
\frac{Q_{\infty}}{Q_{k-j}}=\prod_{\ell \geqslant 1}\left(1-2^{j-k-\ell}\right)=\sum_{m \geqslant 0} \frac{(-1)^{m} 2^{-\left(\begin{array}{c}
m+1 \\
2
\end{array}\right)}}{Q_{m}} 2^{(j-k) m},
$$

which is still valid for $j>k$ (in which case both sides are zero). Substituting the latter into (23) gives

$$
\begin{aligned}
\tilde{M}_{k, 1}(z) & =2^{k} \sum_{0 \leqslant j \leqslant k} \frac{(-1)^{j} 2^{-\left(\begin{array}{c}
j \\
2
\end{array}\right)}}{Q_{j} Q_{k-j}} e^{-2^{j-k} z} \\
& =2^{k} \sum_{j \geqslant 0} \frac{(-1)^{j} 2^{-\left(\begin{array}{c}
j \\
2
\end{array}\right)}}{Q_{j} Q_{\infty}} \sum_{m \geqslant 0} \frac{(-1)^{m} 2^{-\left(\begin{array}{c}
m+1 \\
2
\end{array}\right)+(j-k) m}}{Q_{m}} e^{-2^{j-k} z} \\
& =2^{k} \sum_{m \geqslant 0} \frac{(-1)^{m} 2^{-\left(\begin{array}{c}
m+1 \\
2
\end{array}\right)-k m}}{Q_{m} Q_{\infty}} \sum_{j \geqslant 0} \frac{(-1)^{j} 2^{-\left(\begin{array}{c}
j \\
2
\end{array}\right)+j m}}{Q_{j}} e^{-2^{j-k} z},
\end{aligned}
$$

where interchanging the sums is justified as in the proof of Lemma 1. This proves the lemma since the last series is equal to $(-1)^{m} Q_{\infty} F^{(m)}\left(2^{-k} z\right)$.

From these two lemmas, we get

$$
\tilde{M}_{k, 1}(z)=2^{k} F\left(2^{-k} z\right)+\mathcal{O}(1), \quad(\Re(z) \geqslant 0),
$$

which is the Poissonized version of (2).

The asymptotics of $\mu_{n, k}$ and that of $\tilde{M}_{k}(n)$ can be bridged by the analytic de-Poissonization techniques; see the survey paper [16]. For that purpose, it turns out that the use of JS-admissible functions, a notion introduced in [15], provides more operational flexibility.

Throughout this paper, the generic symbols $\varepsilon, \varepsilon^{\prime}$ always denote small positive quantities whose values are immaterial and not necessarily the same at each occurrence.

Definition 1 ([15]). An entire function $\tilde{f}(z)$ is said to be JS-admissible, denoted by $\tilde{f}(z) \in \mathscr{J} \mathscr{S}$, if the following two conditions hold for $|z| \geqslant 1$.

(I) There exists a constant $\alpha \in \mathbb{R}$ such that $\tilde{f}(z)=\mathcal{O}\left(|z|^{\alpha}\right)$ uniformly for $|\arg (z)| \leqslant \varepsilon$.

(O) Uniformly for $\varepsilon \leqslant|\arg (z)| \leqslant \pi, f(z):=e^{z} \tilde{f}(z)=\mathcal{O}\left(e^{\left(1-\varepsilon^{\prime}\right)|z|}\right)$. [15])

When $\tilde{f} \in \mathscr{J} \mathscr{S}$, its coefficients can be expressed in terms of the Poisson-Charlier expansion (see

$$
n !\left[z^{n}\right] e^{z} \tilde{f}(z)=\sum_{j \geqslant 0} \frac{\tilde{f}^{(j)}(n)}{j !} \tau_{j}(n),
$$

which is not only an identity but also an asymptotic expansion, where the $\tau_{j}(n)$ 's are essentially Charlier polynomials defined by

$$
\tau_{j}(n)=n !\left[t^{n}\right] e^{t}(t-n)^{j}=\sum_{0 \leqslant \ell \leqslant j}\left(\begin{array}{l}
j \\
\ell
\end{array}\right)(-n)^{j-\ell} \frac{n !}{(n-\ell) !}, \quad(j=0,1, \ldots) .
$$

In particular, $\tau_{j}(n)$ is a polynomial in $n$ of degree $\left\lfloor\frac{1}{2} j\right\rfloor$; the expressions for $\tau_{j}(n), 0 \leqslant j \leqslant 5$, are given below. 


$$
\begin{array}{cccccc}
\tau_{0}(n) & \tau_{1}(n) & \tau_{2}(n) & \tau_{3}(n) & \tau_{4}(n) & \tau_{5}(n) \\
\hline 1 & 0 & -n & 2 n & 3 n(n-2) & -4 n(5 n-6)
\end{array}
$$

For our purpose, we also need an additional uniformity property for JS-admissible functions as the level parameter $k$ may also depend on $n$.

Lemma 3. The functions $\tilde{M}_{k, 1}(z)$ are uniformly JS-admissible: if $|z| \geqslant 1$, then for $|\arg (z)| \leqslant \varepsilon$

$$
\tilde{M}_{k, 1}(z)=\mathcal{O}(|z|)
$$

and for $\varepsilon \leqslant|\arg (z)| \leqslant \pi$

$$
e^{z} \tilde{M}_{k, 1}(z)=\mathcal{O}\left(e^{\left(1-\varepsilon^{\prime}\right)|z|}\right),
$$

where all implied constants are absolute and hold uniformly for $k \geqslant 0$.

Proof. Consider first the region $|\arg (z)| \geqslant \varepsilon$. Let $M_{k, 1}(z):=e^{z} \tilde{M}_{k, 1}(z)$. The bound (30) holds trivially for $k=0$ since $M_{0,1}(z)=1$. Thus, we assume $k \geqslant 1$. Then, we can rewrite (20) into the following form

$$
M_{k, 1}(z)=\int_{0}^{z} 2 e^{\frac{1}{2} u} M_{k-1,1}\left(\frac{1}{2} u\right) \mathrm{d} u=2 z \int_{0}^{1} e^{\frac{1}{2} t z} M_{k-1,1}\left(\frac{1}{2} t z\right) \mathrm{d} t .
$$

By the trivial bound $\mu_{n, k} \leqslant 2 n$, we get the a priori upper estimate $\left|M_{k, 1}(z)\right| \leqslant 2|z| e^{|z|}$ (which also holds for $k=0$ ). Plugging this into (31) yields

$$
\begin{aligned}
\left|M_{k, 1}(z)\right| & \leqslant 2|z|^{2} \int_{0}^{1} t e^{\frac{1}{2} t(\Re(z)+|z|)} \mathrm{d} t \\
& \leqslant 2|z|^{2} \int_{0}^{1} e^{\frac{1}{2} t(\cos \varepsilon+1)|z|} \mathrm{d} t \\
& \leqslant \frac{4|z|}{\cos \varepsilon+1} e^{\frac{1}{2}(\cos \varepsilon+1)|z|} .
\end{aligned}
$$

Since $\frac{1}{2}<\frac{1}{2}(\cos \varepsilon+1)<1$, this proves $(30)$.

Now we consider the sector $|\arg (z)| \leqslant \varepsilon$. The required bound $\tilde{M}_{k, 1}(z)=\mathcal{O}(|z|)$ will follow from (28) and the smallness of $F(z) / z$ to be proved in Proposition 1 below (see also Remark 3). It is also possible to give a direct proof, although with a weaker estimate (sufficient for our de-Poissonization purposes, however, which would lead to weaker error terms in the subsequent estimates). By (31)

$$
\tilde{M}_{k, 1}(z)=2 z \int_{0}^{1} e^{-(1-t) z} \tilde{M}_{k-1,1}\left(\frac{1}{2} t z\right) \mathrm{d} t
$$

and induction on $k$, we deduce the (slightly worse) bound $\tilde{M}_{k, 1}(z)=\mathcal{O}\left(|z|^{1+\varepsilon^{\prime}}\right)$.

By the asymptotic expansion (29) and Lemma 3 (which also gives bounds on the derivatives of $\tilde{M}_{k, 1}(z)$ by Ritt's theorem; see [15]), we can justify the "Poisson heuristic" for $\mu_{n, k}$ as follows.

Lemma 4. For large $n$ and $0 \leqslant k \leqslant n$

$$
\mu_{n, k}=\tilde{M}_{k, 1}(n)+\mathcal{O}(1)
$$

where the $\mathcal{O}$-term holds uniformly in $k$.

From this and (28), we obtain now Theorem 1 (except for the positivity of $F(x)$, which will be established in Proposition 2 below). 


\subsection{Asymptotics of $F(z)$}

In this subsection, we derive an asymptotic expansion for $F(z)$ for small $|z|$ and prove the positivity of $F(x)$ on $\mathbb{R}^{+}$; the corresponding large- $|z|$ asymptotics is much easier; see (5).

Proposition 1. For each integer $m \geqslant 0$, the $m$ th derivative of $F$ satisfies

$$
F^{(m)}(z)=\frac{\rho^{m+\frac{1}{2}+\frac{1}{\log 2}}}{\sqrt{2 \pi \log _{2} \rho}} \exp \left(-\frac{(\log \rho)^{2}}{2 \log 2}-P\left(\log _{2} \rho\right)\right)\left(1+\mathcal{O}\left(|\log \rho|^{-1}\right)\right),
$$

as $|z| \rightarrow 0$ in the sector $|\arg (z)| \leqslant \varepsilon$, where $P(t)$ is given in (7) and $\rho=\rho(z)$ solves the saddle-point equation $\frac{\rho}{\log \rho}=\frac{1}{z \log 2}$, and satisfies $|\rho| \rightarrow \infty$ as $|z| \rightarrow 0$.

Proof. By additivity, the Laplace transform of $F$ has the form

$$
\mathscr{L}[F(z) ; s]=\sum_{j \geqslant 0} \frac{(-1)^{j} 2^{-\left(\begin{array}{c}
j \\
2
\end{array}\right)}}{Q_{j} Q_{\infty}\left(s+2^{j}\right)}, \quad(\Re(s)>-1),
$$

which equals the partial fraction expansion of the product

$$
\mathscr{L}[F(z) ; s]=\prod_{j \geqslant 0} \frac{1}{1+2^{-j s}}=\frac{1}{Q(-2 s)} .
$$

Since we are interested in the asymptotics of $F(z)$ as $|z| \rightarrow 0$, which is reflected by the large- $|s|$ asymptotics of $\mathscr{L}[F(z) ; s]$, we apply Mellin transform techniques for that purpose; see Flajolet et al.'s survey paper [12] for more background on tools and applications. In particular, taking the logarithm on both sides of (33) and using the inverse Mellin transform gives

$$
\log Q(-2 s)=\sum_{j \geqslant 0} \log \left(1+2^{-j} s\right)=\frac{1}{2 \pi i} \int_{-\frac{1}{2}-i \infty}^{-\frac{1}{2}+i \infty} \frac{\pi s^{-\omega}}{\left(1-2^{\omega}\right) \omega \sin \pi \omega} \mathrm{d} \omega,
$$

because the Mellin transform of $\log (1+s)$ equals

$$
\int_{0}^{\infty} s^{\omega-1} \log (1+s) \mathrm{d} s=\frac{\pi}{\omega \sin \pi \omega}, \quad(\Re(\omega) \in(-1,0)) .
$$

Now, by standard Mellin analysis (see [12]), we deduce that

$$
\log Q(-2 s)=\frac{(\log s)^{2}}{2 \log 2}+\frac{\log s}{2}+P\left(\log _{2} s\right)+\mathcal{O}\left(|s|^{-1}\right)
$$

uniformly as $|s| \rightarrow \infty$ in the sector $|\arg s| \leqslant \pi-\varepsilon$.

Next, by the inverse Laplace transform, first for $z=x$ real, we have

$$
F^{(m)}(x)=\frac{1}{2 \pi i} \int_{1-i \infty}^{1+i \infty} \frac{s^{m} e^{x s}}{Q(-2 s)} \mathrm{d} s .
$$

It follows, by moving the line of integration to $\Re(s)=\rho$ and by substituting the asymptotic approximation (34), that

$$
F^{(m)}(x)=\frac{1}{2 \pi i} \int_{\rho-i \infty}^{\rho+i \infty} s^{m} \exp \left(x s-\frac{(\log s)^{2}}{2 \log 2}-\frac{\log s}{2}-P\left(\log _{2} s\right)+\mathcal{O}\left(|s|^{-1}\right)\right) \mathrm{d} s .
$$


A standard application of the saddle-point method (see [13, Ch. VIII]) then yields (32) for real $z$ with $z \rightarrow 0$.

When the imaginary part of $z$ is not zero, we can still apply the same procedure but need to deform the integration contour in the representation (35) from the vertical line with real part 1 to the one where the two portions from $1+i$ to $1+i \infty$ and from $1-i \infty$ to $1-i$ are tilted slightly to the left; see Appendix A for details.

Remark 3. As a consequence of the above proposition, we see that $F(z)$ is smaller than any polynomial of $z$ as $|z| \rightarrow 0$ in the sector $|\arg (z)| \leqslant \varepsilon$.

Asymptotically, for large $X:=\frac{1}{x \log 2}, x \in \mathbb{R}$,

$$
\rho=X\left(\log X+\log \log X+\frac{\log \log X}{\log X}-\frac{(\log \log X)^{2}-2 \log \log X}{2(\log X)^{2}}+\mathcal{O}\left(\frac{(\log \log X)^{3}}{(\log X)^{3}}\right)\right) .
$$

Substituting this into (32) gives the more explicit expression (6).

Finally, we prove the positivity of $F$ on the positive real line.

Proposition 2. The function $F(x)$ is positive in $(0, \infty)$.

Proof. Since $\mu_{n, k} \geqslant 0$, we see, by Theorem 1, that $F(x) \geqslant 0$ on $(0, \infty)$. Then, from (33),

$$
(1+s) \mathscr{L}[F(x) ; s]=\prod_{j \geqslant 0} \frac{1}{1+2^{-j-1} s}=\mathscr{L}\left[F(x) ; \frac{1}{2} s\right] .
$$

The corresponding inverse Laplace transform yields the equation $F(x)+F^{\prime}(x)=2 F(2 x)$. With this differential-functional equation, we prove the positivity of $F$ by contradiction. Assume a contrario that $F(x)$ has a zero in $(0, \infty)$, say $x_{0}$. Then $F^{\prime}\left(x_{0}\right)=2 F\left(2 x_{0}\right) \geqslant 0$ and since $F^{\prime}\left(x_{0}\right)>0$ is not possible (for otherwise $F(x)$ would become negative in a neighborhood of $x_{0}$ ), we also have $F\left(2 x_{0}\right)=0$. Continuing this argument, we obtain arbitrarily large zeros. This is, however, impossible since we see from (5) that $F(x)$ is positive for all $x$ large enough.

\section{The Variance of the External Profile}

In this section, we prove Theorem 2 by the same approach used above for the mean, starting from the second moment $\nu_{n, k}:=\mathbb{E}\left(B_{n, k}^{2}\right)$, which satisfies, by (18), the recurrence

$$
\nu_{n, k}=2^{2-n} \sum_{0 \leqslant j<n}\left(\begin{array}{c}
n-1 \\
j
\end{array}\right) \nu_{j, k-1}+2^{2-n} \sum_{0 \leqslant j<n}\left(\begin{array}{c}
n-1 \\
j
\end{array}\right) \mu_{j, k-1} \mu_{n-1-j, k-1}, \quad(n, k \geqslant 1),
$$

with the boundary conditions $\nu_{0,0}=1$, and $\nu_{n, 0}=\nu_{0, k}=0$ for $n, k \geqslant 1$. Translating this recurrence into the corresponding Poisson generating function

$$
\tilde{M}_{k, 2}(z):=e^{-z} \sum_{n \geqslant 0} \nu_{n, k} \frac{z^{n}}{n !}, \quad(k \geqslant 0),
$$

leads to the differential-functional equation

$$
\tilde{M}_{k, 2}(z)+\tilde{M}_{k, 2}^{\prime}(z)=2 \tilde{M}_{k-1,2}\left(\frac{1}{2} z\right)+2 \tilde{M}_{k-1,1}\left(\frac{1}{2} z\right)^{2}, \quad(k \geqslant 1),
$$

with $\tilde{M}_{0,2}(z)=e^{-z}$. 
Since the variance is expected to be of the same order as the mean (notably when both tend to infinity), there is a cancellation between the dominant term in the asymptotic expansion for $\nu_{n, k}$ and that for $\mu_{n, k}^{2}$. Such a cancellation of dominant terms can be more easily manipulated by considering the Poissonized variance (as in $[14,15]$ ):

$$
\tilde{V}_{k}(z)=\tilde{M}_{k, 2}(z)-\tilde{M}_{k, 1}(z)^{2}-z \tilde{M}_{k, 1}^{\prime}(z)^{2},
$$

which itself also satisfies, after a straightforward calculation,

$$
\tilde{V}_{k}(z)+\tilde{V}_{k}^{\prime}(z)=2 \tilde{V}_{k-1}\left(\frac{1}{2} z\right)+z \tilde{M}_{k, 1}^{\prime \prime}(z)^{2}, \quad(k \geqslant 1)
$$

with $\tilde{V}_{0}(z)=e^{-z}-(1+z) e^{-2 z}$. In this form, the original inherent cancellation is nicely integrated into the same type of equation with an explicitly computable non-homogeneous function, and we need only to work out the asymptotics of $\tilde{V}_{k}(z)$, which will be proved to be asymptotically equivalent to the variance of $B_{n, k}$ in the major range of interest.

\subsection{Exact Expressions}

To justify the cancellation-free approach for computing the asymptotic variance, we still need more explicit expressions for $\tilde{M}_{k, 2}(z)$ and $\tilde{V}_{k}(z)$. For that purpose, we apply the Laplace transform on both sides of (37) and obtain

$$
\mathscr{L}\left[\tilde{M}_{k, 2}(z) ; s\right]=\frac{4}{s+1} \mathscr{L}\left[\tilde{M}_{k-1,2}(z) ; 2 s\right]+\frac{4}{s+1} \mathscr{L}\left[\tilde{M}_{k-1,1}(z)^{2} ; 2 s\right], \quad(k \geqslant 1),
$$

with $\mathscr{L}\left[\tilde{M}_{0,2}(z) ; s\right]=\frac{1}{s+1}$. This is solved by iterating the recurrence, giving

$$
\mathscr{L}\left[\tilde{M}_{k, 2}(z) ; s\right]=\frac{4^{k}}{(s+1) \cdots\left(2^{k} s+1\right)}+\sum_{0 \leqslant j<k} \frac{4^{k-j} \mathscr{L}\left[\tilde{M}_{j, 1}(z)^{2} ; 2^{k-j} s\right]}{(s+1) \cdots\left(2^{k-j-1} s+1\right)} .
$$

From (23), a manageable expression for the Laplace transform of $\tilde{M}_{j, 1}(z)^{2}$ is given by

$$
\mathscr{L}\left[\tilde{M}_{j, 1}(z)^{2} ; s\right]=4^{j} \sum_{0 \leqslant h, \ell \leqslant j} \frac{(-1)^{h+\ell} 2^{-\left(\begin{array}{c}
h \\
2
\end{array}\right)-\left(\begin{array}{c}
\ell \\
2
\end{array}\right)}}{Q_{h} Q_{j-h} Q_{\ell} Q_{j-\ell}} \cdot \frac{1}{s+2^{h-j}+2^{\ell-j}} .
$$

This and the partial fraction expansion (22) yield

$$
\begin{aligned}
& \sum_{0 \leqslant j<k} \frac{4^{k-j} \mathscr{L}\left[\tilde{M}_{j, 1}(z)^{2} ; 2^{k-j} s\right]}{(s+1) \cdots\left(2^{k-j-1} s+1\right)} \\
& =\sum_{(j, r, h, \ell) \in \mathscr{S}} \frac{2^{2 j+1}(-1)^{r+h+\ell} 2^{-\left(\begin{array}{c}
r \\
2
\end{array}\right)-\left(\begin{array}{l}
h \\
2
\end{array}\right)-\left(\begin{array}{l}
\ell \\
2
\end{array}\right)}}{Q_{r} Q_{k-1-j-r} Q_{h} Q_{j-h} Q_{\ell} Q_{j-\ell}} \cdot \frac{1}{\left(s+2^{r-k+1+j}\right)\left(s+2^{h-k}+2^{\ell-k}\right)},
\end{aligned}
$$

where

$$
\mathscr{S}=\{(j, r, h, \ell): 0 \leqslant j \leqslant k-1,0 \leqslant r \leqslant k-1-j, 0 \leqslant h, \ell \leqslant j\} .
$$

From this and the expression

$$
\frac{1}{(s+u)(s+v)}=\frac{1}{v-u}\left(\frac{1}{s+u}-\frac{1}{s+v}\right), \quad(u \neq v),
$$


we obtain, by term-by-term inversion,

$$
\tilde{M}_{k, 2}(z)=\tilde{M}_{k, 1}(z)+\sum_{(j, r, h, \ell) \in \mathscr{S}} \frac{2^{2 j+1}(-1)^{r+h+\ell} 2^{-\left(\begin{array}{c}
r \\
2
\end{array}\right)-\left(\begin{array}{c}
h \\
2
\end{array}\right)-\left(\begin{array}{c}
\ell \\
2
\end{array}\right)}}{Q_{r} Q_{k-1-j-r} Q_{h} Q_{j-h} Q_{\ell} Q_{j-\ell}} \phi\left(2^{r-k+1+j}, 2^{h-k}+2^{\ell-k} ; z\right),
$$

where

$$
\phi(u, v ; z)=e^{-v z} \int_{0}^{z} e^{-(u-v) t} \mathrm{~d} t= \begin{cases}\frac{e^{-u z}-e^{-v z}}{v-u}, & \text { if } u \neq v \\ z e^{-u z}, & \text { if } u=v .\end{cases}
$$

Taking the coefficients of $z^{n}$ on both sides, we are led to the exact expression for $\nu_{n, k}$

$$
\nu_{n, k}=\mu_{n, k}+\sum_{(j, r, h, \ell) \in \mathscr{S}} \frac{2^{2 j+1}(-1)^{r+h+\ell_{2}}-\left(\begin{array}{c}
r \\
2
\end{array}\right)-\left(\begin{array}{c}
h \\
2
\end{array}\right)-\left(\begin{array}{c}
\ell \\
2
\end{array}\right)}{Q_{r} Q_{k-1-j-r} Q_{h} Q_{j-h} Q_{\ell} Q_{j-\ell}} \delta\left(2^{r-k+1+j}, 2^{h-k}+2^{\ell-k} ; n\right),
$$

where

$$
\delta(u, v ; n)=n \int_{0}^{1}(1-u-(v-u) t)^{n-1} \mathrm{~d} t= \begin{cases}\frac{(1-u)^{n}-(1-v)^{n}}{v-u}, & \text { if } u \neq v \\ n(1-u)^{n-1}, & \text { if } u=v .\end{cases}
$$

Similarly, by (38) and the same procedure, we also have

$$
\tilde{V}_{k}(z)=\sum_{(j, r, h, \ell) \in \mathscr{V}} \frac{2^{k-j}(-1)^{r+h+\ell_{2}} 2^{-\left(\begin{array}{c}
r \\
2
\end{array}\right)-\left(\begin{array}{c}
h \\
2
\end{array}\right)-\left(\begin{array}{c}
\ell \\
2
\end{array}\right)+2 h+2 \ell}}{Q_{r} Q_{k-j-r} Q_{h} Q_{j-h} Q_{\ell} Q_{j-\ell}} \varphi\left(2^{j+r}, 2^{h}+2^{\ell} ; 2^{-k} z\right),
$$

where

$$
\mathscr{V}=\{(j, r, h, \ell): 0 \leqslant j \leqslant k, 0 \leqslant r \leqslant k-j, 0 \leqslant h, \ell \leqslant j\},
$$

and $\varphi(u, v ; z)$ is defined in (10). Note that the equality $2^{j+r}=2^{h}+2^{\ell}$ occurs if and only if $(j, r, h, \ell)$ belongs to the set

$$
\{(j, r, h, \ell): 1 \leqslant j \leqslant k, r=0, h=\ell=j-1 \quad \text { or } \quad 0 \leqslant j<k, r=1, h=\ell=j\},
$$

and the corresponding terms in (41) are given by

$$
\sum_{1 \leqslant j \leqslant k} \frac{2^{-k-j} 2^{-2\left(\begin{array}{c}
j-1 \\
2
\end{array}\right)+4(j-1)}}{Q_{k-j} Q_{1}^{2} Q_{j-1}^{2}} \cdot \frac{z^{2}}{2} e^{-2^{j-k} z}-\sum_{0 \leqslant j<k} \frac{2^{-k-j} 2^{-2\left(\begin{array}{l}
j \\
2
\end{array}\right)+4 j}}{Q_{k-j-1} Q_{1} Q_{j}^{2}} \cdot \frac{z^{2}}{2} e^{-2^{j+1-k} z},
$$

which becomes zero since $Q_{1}=\frac{1}{2}$. Hence, the equality part in the definition (10) of $\varphi(u, v ; z)$ may be ignored.

\subsection{Asymptotics of $\operatorname{Var}\left(B_{n, k}\right)$}

The range where $2^{k} n e^{-2^{-k} n} \rightarrow 0$ can be treated elementarily, as in the case of the mean. In this range of $k$ (and even in the wider range where $2^{-k} n \rightarrow \infty$ ), we have

$$
\operatorname{Var}\left(B_{n, k}\right) \sim \frac{2^{k}}{Q_{k}}\left(1-2^{-k}\right)^{n},
$$

uniformly in $k$. To prove this, we use (40) and begin with the estimate

$$
\delta\left(2^{r-k+1+j}, 2^{h-k}+2^{\ell-k} ; n\right)=\mathcal{O}\left(n\left(1-2^{1-k}\right)^{n}\right),
$$


where the implied constant is absolute in $n$ and in $k$. Substituting this into (40) yields

$$
\begin{aligned}
\nu_{n, k} & =\mu_{n, k}+\mathcal{O}\left(n\left(1-2^{1-k}\right)^{n} \sum_{(j, r, h, \ell) \in \mathscr{S}} 2^{2 j} 2^{-\left(\begin{array}{c}
r \\
2
\end{array}\right)-\left(\begin{array}{c}
h \\
2
\end{array}\right)-\left(\begin{array}{c}
\ell \\
2
\end{array}\right)}\right) \\
& =\mu_{n, k}+\mathcal{O}\left(4^{k} n\left(1-2^{1-k}\right)^{n}\right) .
\end{aligned}
$$

By the asymptotic estimate (4) for $\mu_{n, k}$, we then have

$$
\nu_{n, k}=\frac{2^{k}}{Q_{k}}\left(1-2^{-k}\right)^{n}\left(1+\mathcal{O}\left(e^{-\frac{n}{2^{k}-1}}+2^{k} n \frac{\left(1-2^{1-k}\right)^{n}}{\left(1-2^{-k}\right)^{n}}\right)\right) .
$$

From this estimate and (26), we see that

$$
\nu_{n, k} \sim \frac{2^{k}}{Q_{k}}\left(1-2^{-k}\right)^{n} \sim \mu_{n, k},
$$

because $2^{k} n e^{-2^{-k} n} \rightarrow 0$. Also, $\mu_{n, k}=o(1)$ in this range. Thus, we get $\mu_{n, k}^{2}=o\left(\mu_{n, k}\right)$ and then (42). Note that it is possible to extend slightly the range to $2^{k} e^{-2^{-k} n} \rightarrow 0$ because there is only one term containing the factor $n\left(1-2^{1-k}\right)^{n}$ in the sum (40) (which is when $j=r=h=l=0$ ), and the contribution of all other terms is bounded above by $\mathcal{O}\left(4^{k}\left(1-2^{1-k}\right)^{n}\right)$. Moreover, that (42) holds in the wider range $2^{-k} n \rightarrow \infty$ follows from a refinement of the expansion of Theorem 2 , which can in turn be obtained by the analytic method below.

On the other hand, complex analytic tools apply in a wider range. In contrast to the mean, however, we do not prove an identity for $\tilde{V}_{k}(z)$ (compare with Lemma 2 and see Remark 4) but we directly prove an asymptotic result similar to the one for the mean in (28).

Lemma 5. For $|\arg (z)| \leqslant \varepsilon$ and $k \geqslant 0, \tilde{V}_{k}(z)$ satisfies the expansion

$$
\tilde{V}_{k}(z)=2^{k} G\left(2^{-k} z\right)+\mathcal{O}(1),
$$

where $G(z)$ is defined in Theorem 2.

Proof. Similar to Lemma 2, we first consider $Q_{\infty} / Q_{k-j-r}$, which satisfies the uniform bound

$$
\frac{Q_{\infty}}{Q_{k-j-r}}=\prod_{\ell \geqslant 1}\left(1-2^{j+r-k-\ell}\right)=1+\mathcal{O}\left(2^{j+r-k}\right) .
$$

Here the product also makes sense for $j+r>k$ where it becomes zero and thus the bound also holds in this case. Substituting this into (41) gives

$$
\tilde{V}_{k}(z)=2^{k} G\left(2^{-k} z\right)+\sum_{j, r \geqslant 0} \sum_{0 \leqslant h, \ell \leqslant j} \frac{2^{-j-\left(\begin{array}{c}
r \\
2
\end{array}\right)-\left(\begin{array}{c}
h \\
2
\end{array}\right)-\left(\begin{array}{c}
\ell \\
2
\end{array}\right)+2 h+2 \ell}}{Q_{\infty} Q_{r} Q_{h} Q_{j-h} Q_{\ell} Q_{j-\ell}} \mathcal{O}\left(2^{j+r} \varphi\left(2^{j+r}, 2^{h}+2^{\ell} ; 2^{-k} z\right)\right) .
$$

To estimate the double sum, we split the summation range into two: (i) $h, \ell \leqslant\lfloor j / 2\rfloor$ and (ii) either $h>\lfloor j / 2\rfloor$ or $\ell>\lfloor j / 2\rfloor$, and denote the resulting sums by $E_{1}(z)$ and $E_{2}(z)$, respectively. By the estimates

$$
2^{j+r} \varphi\left(2^{j+r}, 2^{h}+2^{\ell} ; 2^{-k} z\right)= \begin{cases}\mathcal{O}(1), & \text { if } h, \ell \leqslant\lfloor j / 2\rfloor, \\ \mathcal{O}\left(2^{j+r}\right), & \text { if } h>\lfloor j / 2\rfloor \text { or } \ell>\lfloor j / 2\rfloor,\end{cases}
$$

where the implied constants are both absolute, we have

$$
E_{1}(z)=\mathcal{O}\left(\sum_{j, r \geqslant 0} \sum_{0 \leqslant h, \ell \leqslant j / 2} 2^{-j-\left(\begin{array}{c}
r \\
2
\end{array}\right)-\left(\begin{array}{l}
h \\
2
\end{array}\right)-\left(\begin{array}{l}
\ell \\
2
\end{array}\right)+2 h+2 \ell}\right)=\mathcal{O}(1),
$$


and

$$
\begin{aligned}
E_{2}(z) & =\mathcal{O}\left(\sum_{j, r \geqslant 0} \sum_{\substack{0 \leqslant h, \ell \leqslant j \\
h>j / 2 \text { or } \ell>j / 2}} 2^{-\left(\begin{array}{c}
r \\
2
\end{array}\right)-\left(\begin{array}{c}
h \\
2
\end{array}\right)-\left(\begin{array}{l}
\ell \\
2
\end{array}\right)+r+2 h+2 \ell}\right) \\
& =\mathcal{O}\left(\sum_{j \geqslant 1} j^{-1} 2^{-\frac{1}{8} j^{2}+\frac{5}{4} j}\right)=\mathcal{O}(1) .
\end{aligned}
$$

This proves the claimed expansion.

Remark 4. Comparing with Lemma 2 , it would be natural to derive an identity for $\tilde{V}_{k}(z)$ in a way similar to that for $\tilde{M}_{k, 1}(z)$ (see Lemma 2) by replacing the first order asymptotics (44) by the full expansion

$$
\frac{Q_{\infty}}{Q_{k-j-r}}=\prod_{\ell \geqslant 1}\left(1-2^{j+r-k-\ell}\right)=\sum_{m \geqslant 0} \frac{(-1)^{m} 2^{-\left(\begin{array}{c}
m+1 \\
2
\end{array}\right)}}{Q_{m}} 2^{(j+r-k) m}
$$

which is zero for $j+r>k$. However, doing so yields an expression that is no more absolutely convergent, as pointed out by one referee.

Nevertheless, ignoring the convergence issue and carrying out all computations formally, we can expand $\tilde{V}_{k}(z)$ as

$$
\tilde{V}_{k}(z)=2^{k} \sum_{m \geqslant 0} \frac{2^{-\left(\begin{array}{c}
m+1 \\
2
\end{array}\right)-m k}}{Q_{m}} H_{m}\left(2^{-k} z\right),
$$

where $H_{m}(z)$ are suitable functions. Then, a formal calculation of the Laplace transform gives (after a lengthy computation)

$$
\mathscr{L}\left[H_{m}(z) ; s\right]=s^{m} \sum_{j \geqslant 0} 4^{-j} \frac{\tilde{g}_{j}^{*}\left(2^{-j} s\right)}{Q\left(-2^{1-j} s\right)},
$$

where $\tilde{g}_{j}^{*}(s)=\mathscr{L}\left[z\left(\tilde{M}_{j, 1}^{\prime \prime}(z)\right)^{2} ; s\right]$. Similarly,

$$
\mathscr{L}\left[G^{(m)}(z) ; s\right]=s^{m} \sum_{j \geqslant 0} 4^{-j} \frac{\tilde{g}_{j}^{*}\left(2^{-j} s\right)}{Q\left(-2^{1-j} s\right)},
$$

where this relation indeed holds not just formally but also in a rigorous analytic sense; see Appendix B for a proof. This will be needed in the next subsection.

Now, (46) and (47) suggest that $H_{m}(z)=G^{(m)}(z)$ for $m \geqslant 0$, which in turn suggests the validity of the following identity

$$
\tilde{V}_{k}(z)=2^{k} \sum_{m \geqslant 0} \frac{2^{-\left(\begin{array}{c}
m+1 \\
2
\end{array}\right)-k m}}{Q_{m}} G^{(m)}\left(2^{-k} z\right) .
$$

This identity was claimed in the conference version of this paper, but is not needed for our purposes here.

Note that (43) gives the version of (8) under the Poisson model. From this we deduce Theorem 8 by the same approaches used to prove Theorem 1, namely, de-Poissonization techniques through the use of JS-admissible functions.

Lemma 6. The functions $\tilde{M}_{k, 2}(z)$ are uniformly JS-admissible. More precisely, if $|z| \geqslant 1$, then for $|\arg (z)| \leqslant \varepsilon$

$$
\tilde{M}_{k, 2}(z)=\mathcal{O}\left(|z|^{2}\right)
$$


and for $\varepsilon \leqslant|\arg (z)| \leqslant \pi$

$$
e^{z} \tilde{M}_{k, 2}(z)=\mathcal{O}\left(e^{\left(1-\varepsilon^{\prime}\right)|z|}\right)
$$

where all implied constants in the $\mathcal{O}$-terms are absolute for $k \geqslant 0$.

Proof. We proved in [15, Prop. 2.4] that if $\tilde{g}(z)$ is JS-admissible, then $\tilde{f}(z)$ with $\tilde{f}(0)=0$ and

$$
\tilde{f}(z)+\tilde{f}^{\prime}(z)=2 \tilde{f}\left(\frac{1}{2} z\right)+\tilde{g}(z),
$$

is also JS-admissible. Since $2 \tilde{M}_{k-1,1}\left(\frac{1}{2} z\right)^{2}$ is uniformly JS-admissible (by Lemma 3 ), the same property holds for $\tilde{M}_{k, 2}(z)$ by the same proof of [15, Prop. 2.4].

We are now ready to prove Theorem 2 . Since $\tilde{M}_{k, 2} \in \mathscr{J} \mathscr{S}$, we have the expansion

$$
\nu_{n, k}=\sum_{0 \leqslant j \leqslant 2} \frac{\tilde{M}_{k, 2}^{(j)}(n)}{j !} \tau_{j}(n)+\mathcal{O}(1),
$$

where we retain the terms from (29) with $j \leqslant 2$ so as to guarantee that the error term is $\mathcal{O}(1)$ (here we used estimates for the derivative of $\tilde{M}_{k, 2}(z)$ which follow from (48) and Ritt's theorem to bound the error term). For $\mu_{n, k}$, we need an expansion with an error up to $\mathcal{O}\left(n^{-1}\right)$ :

$$
\mu_{n, k}=\sum_{0 \leqslant j \leqslant 2} \frac{\tilde{M}_{k, 1}^{(j)}(n)}{j !} \tau_{j}(n)+\mathcal{O}\left(n^{-1}\right),
$$

so that $\mu_{n, k}^{2}$ is correct up to an error of $\operatorname{order} \mathcal{O}(1)$. Then we obtain

$$
\operatorname{Var}\left(B_{n, k}\right)=\nu_{n, k}-\mu_{n, k}^{2}=\tilde{V}_{k}(n)-\frac{n}{2} \tilde{V}_{k}^{\prime \prime}(n)+\mathcal{O}(1),
$$

where we have used the relation $\tilde{M}_{k, 2}(n)=\tilde{V}_{k}(n)+\tilde{M}_{k, 1}(n)^{2}+n \tilde{M}_{k, 1}^{\prime}(n)^{2}$. Now, from (43), we have

$$
\tilde{V}_{k}^{\prime \prime}(n)=2^{-k} G^{\prime \prime}\left(2^{-k} n\right)+\mathcal{O}\left(n^{-2}\right)=\mathcal{O}\left(n^{-1}\right),
$$

where the last estimate follows from properties of $G(z)$; see the next subsection. Plugging this and the expansion from (43) into (49) gives the approximation in Theorem 2.

\subsection{Asymptotics of $G(z)$}

We now derive the asymptotic behaviors of $G$ for small and large $|z|$, and prove that $G(x)$ is positive for $x \in(0, \infty)$. In particular, the asymptotic approximations of $G$ will imply (11).

First, the asymptotics of $G(z)$ for large $z$ follows directly from the defining series (9):

$$
G(z)=\frac{e^{-z}}{Q_{\infty}}+\mathcal{O}\left(|z| e^{-2 \Re(z)}\right)
$$

for $\Re(z)>0$; see (12) which is the real-valued version of this asymptotics. Note that similar expansions can also be derived for the derivatives of $G(z)$.

In contrast, the small-| $|z|$ asymptotics of $G$ which we now examine turns out to be very involved. For the sake of simplicity, we restrict ourselves only to the real case since this suffices for our applications.

Proposition 3. For each integer $m \geqslant 0, G^{(m)}$ satisfies the asymptotic estimate

$$
G^{(m)}(x) \sim 2 F^{(m)}(x),
$$

as $x \rightarrow 0$. 
The proof of this proposition is long and technical and relies mostly on the Laplace transform. Note that since the Laplace transform of $G^{(m)}$ is just $s^{m}$ times the Laplace transform of $G$, it suffices to consider only the case $m=0$.

We start from the Laplace transform of $G(x)$, which, by (47), is given by

$$
\mathscr{L}[G(x) ; s]=\sum_{j \geqslant 0} R_{j}(s), \quad \text { where } \quad R_{j}(s):=\frac{\tilde{g}_{j}^{*}\left(2^{-j} s\right)}{4^{j} Q\left(-2^{1-j} s\right)} .
$$

Here $\tilde{g}_{j}^{*}(s)=\mathscr{L}\left[z\left(\tilde{M}_{j, 1}^{\prime \prime}(z)\right)^{2} ; s\right]$, which, by (23) and a straightforward computation, has the form

$$
\tilde{g}_{j}^{*}(s)=4^{-j} \sum_{0 \leqslant h, \ell \leqslant j} \frac{(-1)^{h+\ell} 2^{-\left(\begin{array}{c}
h \\
2
\end{array}\right)-\left(\begin{array}{c}
\ell \\
2
\end{array}\right)+2 h+2 \ell}}{Q_{h} Q_{j-h} Q_{\ell} Q_{j-\ell}} \cdot \frac{1}{\left(s+2^{h-j}+2^{\ell-j}\right)^{2}} ;
$$

see also Appendix B.

Surprisingly, the dominating term in (50) is $R_{2}(s) \sim 2 / Q(-2 s)$ for large $|s|$, and the hard part of the analysis consists in showing that $\sum_{j \neq 2} R_{j}(s)=\mathcal{O}\left(\left|R_{2}(s) / s\right|\right)$.

Lemma 7. For large $|s|$ in the half-plane $\Re(s)>0, R_{0}$ and $R_{1}$ satisfy

$$
R_{0}(s)=\frac{s^{-2}}{Q(-2 s)}\left(1+\mathcal{O}\left(|s|^{-1}\right)\right) \quad \text { and } \quad R_{1}(s)=\frac{9 s^{-1}}{Q(-2 s)}\left(1+\mathcal{O}\left(|s|^{-1}\right)\right),
$$

respectively, and $R_{j}$ with fixed $j \geqslant 2$ satisfies

$$
R_{j}(s)=\frac{(2 j-3) ! 2^{\left(\begin{array}{c}
j \\
2
\end{array}\right)}}{((j-2) !)^{2}} \cdot \frac{s^{2-j}}{Q(-2 s)}\left(1+\mathcal{O}\left(|s|^{-1}\right)\right) .
$$

Proof. The estimates (51) for $j=0$ and $j=1$ follow from the closed-form expressions

$$
\tilde{g}_{0}^{*}(s)=\frac{1}{(s+2)^{2}} \quad \text { and } \quad \tilde{g}_{1}^{*}\left(2^{-1} s\right)=\frac{4}{(s+2)^{2}}-\frac{32}{(s+3)^{2}}+\frac{64}{(s+4)^{2}},
$$

and the functional relation $Q(-2 s)=(1+s) Q(-s)$. Thus we assume now $j \geqslant 2$.

Since $\tilde{g}_{j}^{*}\left(2^{-j} s\right)$ is the Laplace transform of $4^{j} z \tilde{M}_{j, 1}^{\prime \prime}\left(2^{j} z\right)^{2}$, we see that the large- $|s|$ behavior of the former is reflected from the small- $|z|$ behavior of the latter. Starting from (21) using Ritt's theorem for the asymptotics of the derivatives of an analytic function (see [13, §VI. 10.1]), we obtain successively the estimates in the following table.

\begin{tabular}{c|ccc}
$f(z)$ & $\tilde{M}_{j, 1}(z)$ & $\tilde{M}_{j, 1}^{\prime \prime}(z)$ & $4^{j} z \tilde{M}_{j, 1}^{\prime \prime}\left(2^{j} z\right)^{2}$ \\
\hline as $|z| \sim 0$ & $\frac{z^{j}}{j ! 2^{j(j-3) / 2}}$ & $\frac{z^{j-2}}{(j-2) ! 2^{j(j-3) / 2}}$ & $\frac{2^{j(j+1)}}{((j-2) !)^{2}} z^{2 j-3}$ \\
\hline \hline $\mathscr{L}[f(z) ; s]$ & $\frac{4^{j}}{\prod_{0 \leqslant \ell \leqslant j}\left(2^{\ell} s+1\right)}$ & $\frac{4^{j} s^{2}}{\prod_{0 \leqslant \ell \leqslant j}\left(2^{\ell} s+1\right)}$ & $\tilde{g}_{j}^{*}\left(2^{-j} s\right)$ \\
\hline as $|s| \rightarrow \infty$ & $2^{-j(j-3) / 2} s^{-j-1}$ & $2^{-j(j-3) / 2} s^{-j+1}$ & $\frac{(2 j-3) !}{((j-2) !)^{2}} 2^{j(j+1)} s^{-2 j+2}$
\end{tabular}

where the entries in the second and the fourth rows give the asymptotics of $f$ and its Laplace transform as $|z| \rightarrow 0$ and $|s| \rightarrow \infty$, respectively. All error terms are of the form $1+\mathcal{O}(|z|)$ and $1+\mathcal{O}\left(|s|^{-1}\right)$, respectively,

From this table and the estimate

$$
\begin{aligned}
Q\left(-2^{1-j} s\right) & =\frac{Q(-2 s)}{(1+s)\left(1+2^{-1} s\right) \cdots\left(1+2^{-(j-1)} s\right)} \\
& =s^{-j} Q(-2 s) 2^{\left(\begin{array}{l}
j \\
2
\end{array}\right)}\left(1+\mathcal{O}\left(|s|^{-1}\right)\right),
\end{aligned}
$$

for large $|s|$, we obtain (52). 
The error term in (52) can be further refined

$$
R_{j}(s)=\frac{(2 j-3) ! 2^{\left(\begin{array}{c}
j \\
2
\end{array}\right)}}{((j-2) !)^{2}} \cdot \frac{s^{2-j}}{Q(-2 s)}\left(1-\frac{7 \cdot 2^{j}-3}{s}+\mathcal{O}\left(4^{j}|s|^{-2}\right)\right)
$$

where the $O$-term being uniform for $j=O(\log |s|)$. From this, we expect that, for large $j$, the bound

$$
R_{j}(s)=\mathcal{O}\left(\frac{\sqrt{j} 2^{j(j+3) / 2}|s|^{-j+2}}{|Q(-2 s)|}\right)
$$

holds uniformly for $j=\mathcal{O}(\log |s|)$. We give a crude version of this in the following lemma, which also incorporates the dependence of $j$ on $|s|$.

Lemma 8. Uniformly for large $|s|$ in the half-plane $\Re(s)>0$,

$$
\tilde{g}_{j}^{*}\left(2^{-j} s\right)= \begin{cases}\mathcal{O}\left(\frac{j|s|^{5}}{\sqrt{\log _{2}|s|}} 2^{-\left(\log _{2}|s|\right)^{2}}\right), & \text { if } j \geqslant \log _{2}|s|-1 \\ \mathcal{O}\left(\sqrt{j} 2^{j^{2}+3 j}|s|^{-2 j+2}\right), & \text { if } 2 \leqslant j \leqslant \log _{2}|s|-1 .\end{cases}
$$

Proof. For notational convenience, we write the Laplace transform of $f$ as $f^{*}$ and that of the product $f_{1} f_{2}$ as the convolution

$$
\left(f_{1}^{*} \star f_{2}^{*}\right)(s):=\left(f_{1} \cdot f_{2}\right)^{*}(s)=\frac{1}{2 \pi i} \int_{\frac{1}{2} s-i \infty}^{\frac{1}{2} s+i \infty} f_{1}^{*}(t) f_{2}^{*}(s-t) \mathrm{d} t .
$$

Since $\tilde{g}_{j}^{*}\left(2^{-j} s\right)=\mathscr{L}\left[4^{j} z \tilde{M}_{j, 1}^{\prime \prime}\left(2^{j} z\right)^{2} ; s\right]$, we see that, by the relation $\mathscr{L}[z f(z) ; s]=-f^{*}(s)^{\prime}$,

$$
\tilde{g}_{j}^{*}\left(2^{-j} s\right)=-\left(L_{j} \star L_{j}\right)^{\prime}(s)=-\left(L_{j} \star L_{j}^{\prime}\right)(s),
$$

where, by (21),

$$
L_{j}(s):=\mathscr{L}\left[2^{j} \tilde{M}_{j, 1}^{\prime \prime}\left(2^{j} z\right) ; s\right]=\frac{s^{2}}{\prod_{0 \leqslant \ell \leqslant j}\left(1+2^{-\ell} s\right)} .
$$

On the other hand, since

$$
L_{j}^{\prime}(s)=L_{j}(s)\left(\frac{2}{s}-\sum_{0 \leqslant \ell \leqslant j} \frac{1}{s+2^{\ell}}\right),
$$

we derive an upper bound for each of the convolutions

$$
\left(L_{j} \star \frac{L_{j}}{s}\right)(s) \quad \text { and } \quad\left(L_{j} \star \frac{L_{j}}{s+2^{\ell}}\right)(s), \quad \text { for } \quad 0 \leqslant \ell \leqslant j .
$$

Note that if both $f_{1}^{*}(s), f_{2}^{*}(s)=\mathcal{O}\left(|s|^{-1}\right)$ for large $|s|$, then we can change the integration path as

$$
\left(f_{1}^{*} \star f_{2}^{*}\right)(s)=\frac{1}{2 \pi i} \int_{\gamma(s)} f_{1}^{*}(t) f_{2}^{*}(s-t) \mathrm{d} t,
$$

where $\gamma(s):=\left\{\frac{1}{2} s(1+i v):-\infty<v<\infty\right\}$ is the symmetry line between 0 and $s$; such a choice implies $|t|=|s-t|$ for $t \in \gamma(s)$ and simplifies our analysis.

If $|s| \geqslant 2^{j+1}$, then $2^{-\ell-1}|s| \geqslant 1$ for $0 \leqslant \ell \leqslant j$, and we have, for $0 \leqslant y \leqslant 2^{j}$,

$$
\frac{1}{2 \pi i} \int_{\gamma(s)} \frac{L_{j}(t) L_{j}(s-t)}{t+y} \mathrm{~d} t=\frac{(s / 2)^{5}}{2 \pi} \int_{-\infty}^{\infty} \frac{\left(1+v^{2}\right)^{2}}{\frac{s}{2}(1+i v)+y} \prod_{0 \leqslant \ell \leqslant j} \frac{1}{1+2^{-\ell} s+4^{-\ell-1} s^{2}\left(1+v^{2}\right)} \mathrm{d} v .
$$


By the upper bound (since $2^{-\ell-1}|s| \geqslant 1$ )

$$
\prod_{0 \leqslant \ell \leqslant j} \frac{1}{1+2^{-\ell} s+4^{-\ell-1} s^{2}\left(1+v^{2}\right)}=\mathcal{O}\left(2^{(j+1)(j+2)}|s|^{-2 j-2}\left(1+v^{2}\right)^{-j-1}\right),
$$

we then obtain

$$
\left|\left(L_{j} \star \frac{L_{j}}{s+y}\right)(s)\right|=\mathcal{O}\left(2^{j^{2}+3 j}|s|^{-2 j+2} \int_{0}^{\infty}\left(1+v^{2}\right)^{-j+1 / 2} \mathrm{~d} v\right)=\mathcal{O}\left(j^{-1 / 2} 2^{j^{2}+3 j}|s|^{-2 j+2}\right) .
$$

Thus, summing over $y=0$ and $y=2^{\ell}, \ell=0,1, \ldots, j$, we have

$$
\tilde{g}_{j}^{*}\left(2^{-j} s\right)=\mathcal{O}\left(\sqrt{j} 2^{j^{2}+3 j}|s|^{-2 j+2}\right),
$$

when $|s| \geqslant 2^{j+1}$, which gives (54) in the small $j$ case.

On the other hand, when $1 \leqslant|s| \leqslant 2^{j+1}$, or $j \geqslant \log _{2}|s|-1$, we have the bounds

$$
\prod_{0 \leqslant \ell \leqslant j}\left(1+2^{-\ell} s\right)=\frac{Q(-2 s)}{\prod_{\ell>j}\left(1+2^{-\ell} s\right)}=\Omega(|Q(-2 s)|),
$$

because

$$
\prod_{\ell>j}\left(1+2^{-\ell} s\right)=\mathcal{O}\left(\prod_{\ell \geqslant j+1}\left(1+2^{-\ell+j+1}\right)\right)=\mathcal{O}(1) .
$$

It follows that, by (34), uniformly as $|s| \rightarrow \infty$ in $|\arg s| \leqslant \pi-\varepsilon$ and $j \geqslant \log _{2}|s|-1$,

$$
L_{j}(s)=\mathcal{O}\left(|s|^{3 / 2} e^{-\Re(\log s)^{2} /(2 \log 2)}\right)=\mathcal{O}\left(|s|^{3 / 2} 2^{-\Re\left(\log _{2} s\right)^{2} / 2}\right) .
$$

Consequently,

$$
\begin{aligned}
\frac{1}{2 \pi i} \int_{\gamma(s)} \frac{L_{j}(t) L_{j}(s-t)}{t+y} \mathrm{~d} t & =\mathcal{O}\left(|s|^{3} 2^{-\Re\left(\log _{2}(s / 2)^{2}\right)} \int_{0}^{\infty}\left(1+v^{2}\right)^{3 / 2-\Re\left(\log _{2}(s / 2)\right)} 2^{-\frac{1}{4} \log _{2}\left(1+v^{2}\right)^{2}} \mathrm{~d} v\right) \\
& =\mathcal{O}\left(\frac{|s|^{5} 2^{-\left(\log _{2}|s|\right)^{2}}}{\sqrt{\log _{2}|s|}}\right) .
\end{aligned}
$$

The first estimate in (54) then follows.

We now derive a precise asymptotics for $\mathscr{L}[G(z) ; s]$ for large $|s|$.

Lemma 9. The Laplace transform of $G$ satisfies

$$
\mathscr{L}[G(z) ; s]=\frac{2}{Q(-2 s)}\left(1+\mathcal{O}\left(|s|^{-1}\right)\right),
$$

as $|s| \rightarrow \infty$ in the half-plane $\Re(s)>0$.

Proof. By Lemma 7, we have $\left(R_{j}(s)\right.$ being defined in (50))

$$
R_{0}(s)+R_{1}(s)+R_{2}(s)=\frac{2}{Q(-2 s)}\left(1+\mathcal{O}\left(|s|^{-1}\right)\right) .
$$

For the remaining terms, we examine the factor $Q\left(-2^{1-j} s\right)$. By (53), we have, uniformly in the half plane $\Re(s)>0$,

$$
Q\left(-2^{1-j} s\right)= \begin{cases}\Omega(1), & \text { if } j \geqslant \log _{2}|s|-1 \\
\Omega\left(|s|^{-j}|Q(-2 s)| 2^{\left(\begin{array}{l}
j \\
2
\end{array}\right)}\right), & \text { if } 0 \leqslant j \leqslant \log _{2}|s| .\end{cases}
$$


Now, it follows from Lemma 8 that

$$
\begin{aligned}
\sum_{3 \leqslant j \leqslant \log _{2}|s|-1} R_{j}(s) & =\mathcal{O}\left(\frac{1}{|Q(-2 s)|} \sum_{3 \leqslant j \leqslant \log _{2}|s|-1} \sqrt{j} 2^{\frac{1}{2} j(j+3)-(j-2) \log _{2}|s|}\right) \\
& =\mathcal{O}\left(\frac{1}{|s Q(-2 s)|}\right) ;
\end{aligned}
$$

also by Lemma 8 and (34)

$$
\begin{aligned}
\sum_{j \geqslant \log _{2}|s|-1} R_{j}(s) & =\mathcal{O}\left(\sum_{j \geqslant \log _{2}|s|-1} \frac{j|s|^{5}}{\sqrt{\log _{2}|s|}} 2^{-\left(\log _{2}|s|\right)^{2}-2 j}\right) \\
& =\mathcal{O}\left(\sqrt{\log _{2}|s|}|s|^{3} 2^{-\left(\log _{2}|s|\right)^{2}}\right) \\
& =\mathcal{O}\left(\frac{\sqrt{\log _{2}|s|}|s|^{7 / 2} 2^{-\left(\log _{2}|s|\right)^{2} / 2}}{|Q(-2 s)|}\right) \\
& =\mathcal{O}\left(\frac{1}{|s Q(-2 s)|}\right),
\end{aligned}
$$

which completes the proof of the lemma.

Proof of Proposition 3. Proposition 3 now follows from Lemma 9 because $\frac{1}{Q(-2 s)}$ is the Laplace transform of $F(z)$, details being similar to the proof of Proposition 1 (for the asymptotics of $F(z)$ ).

Finally, we prove that $G(x)$ is a positive function.

Proposition 4. The function $G(x)$ is positive on $(0, \infty)$.

Proof. By (50) and the inverse Laplace transform, we see that

$$
G(x)=\sum_{j \geqslant 0} 2^{j} \int_{0}^{x}(x-t) \tilde{M}_{j, 1}^{\prime \prime}\left(2^{j}(x-t)\right)^{2} F\left(2^{j} t\right) \mathrm{d} t .
$$

Since $F(x)$ is positive on $(0, \infty)$, we then deduce that $G(x)$ is also positive on $(0, \infty)$.

\section{Internal Profile}

In this section, we present the results without proofs for the internal profile $I_{n, k}$ because all proofs used for the external profile extend to those for the internal profile, which satisfies the same form of recurrence as $B_{n, k}$, namely,

$$
I_{n, k} \stackrel{d}{=} I_{J_{n}, k-1}+I_{n-1-J_{n}, k-1}^{*}, \quad(n, k \geqslant 1),
$$

where the notation is as in (18). The only differences lie in the boundary conditions: $I_{0, k}=0$ for $k \geqslant 0$ and $I_{n, 0}=1$ for $n \geqslant 1$.

From this recurrence and the same method used in Section 2, we can derive the following (mostly known) result for the mean; see [9, 24].

Theorem 4. The expected internal profile satisfies

$$
\mathbb{E}\left(I_{n, k}\right)=2^{k} F_{I}\left(2^{-k} n\right)+\mathcal{O}(1),
$$

uniformly for $0 \leqslant k<n$, where $F_{I}(x)$ equals the following antiderivative of $F(x)$ :

$$
F_{I}(x)=1-\sum_{j \geqslant 0} \frac{(-1)^{j} 2^{-\left(\begin{array}{c}
j+1 \\
2
\end{array}\right)}}{Q_{j} Q_{\infty}} e^{-2^{j} x} .
$$

Moreover, $F_{I}(x)$ is a positive function on $\mathbb{R}^{+}$; see Figure 3 for a plot. 
From the above expression, we see that as $x \rightarrow \infty$

$$
F_{I}(x)=1-\frac{e^{-x}}{Q_{\infty}}+\mathcal{O}\left(e^{-2 x}\right),
$$

the behavior of $F_{I}(x)$ as $x \rightarrow 0$ is the same as (32) with $m=-1$ (by the same analysis given there).
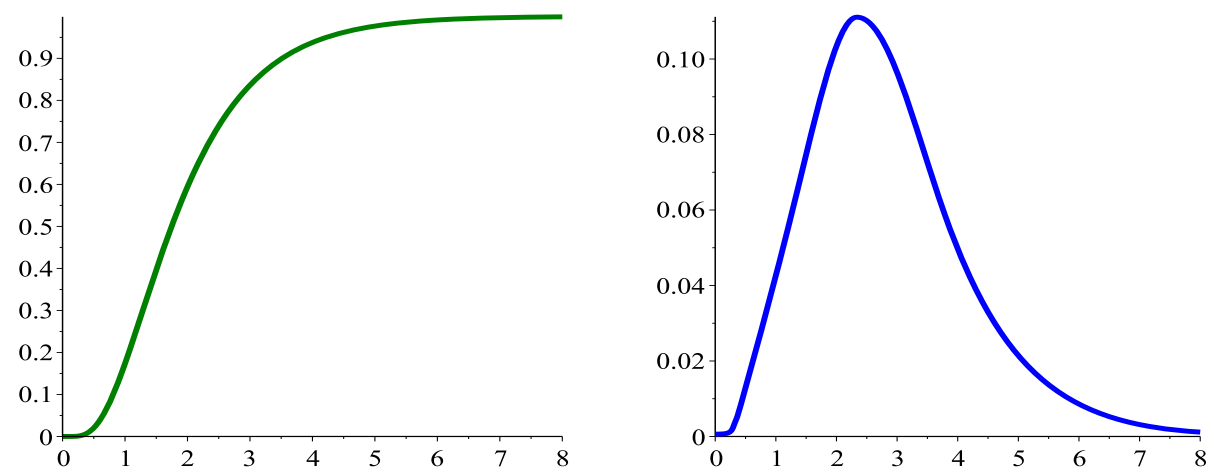

Figure 3: The functions $F_{I}$ (left) and $G_{I}$ (right).

Similarly, the same approaches in Section 3 leads to the following asymptotic expansion for the variance.

Theorem 5. The variance of the internal profile satisfies

$$
\operatorname{Var}\left(I_{n, k}\right)=2^{k} G_{I}\left(2^{-k} n\right)+\mathcal{O}(1),
$$

uniformly for $0 \leqslant k<n$, where $G_{I}(x)$ is positive on $\mathbb{R}^{+}$defined by

$$
G_{I}(x)=\sum_{j, r \geqslant 0} \sum_{0 \leqslant h, \ell \leqslant j} \frac{(-1)^{r+h+\ell_{2}-j-\left(\begin{array}{c}
r \\
2
\end{array}\right)-\left(\begin{array}{c}
h \\
2
\end{array}\right)-\left(\begin{array}{c}
\ell \\
2
\end{array}\right)+h+\ell}}{Q_{\infty} Q_{r} Q_{h} Q_{j-h} Q_{\ell} Q_{j-\ell}} \varphi\left(2^{j+r}, 2^{h}+2^{l} ; x\right),
$$

where $\varphi$ is defined in (10).

Note that the only difference between $G(x)$ and $G_{I}(x)$ is that the exponent $2 h+2 \ell$ in the series definition (9) of $G(x)$ is replaced by $h+\ell$; see Figure 3.

Proposition 5. The function $G_{I}(x)$ satisfies

$$
G_{I}(x) \sim \begin{cases}\frac{e^{-x}}{Q_{\infty}}, & \text { if } x \rightarrow \infty \\ F_{I}(x), & \text { if } x \rightarrow 0 .\end{cases}
$$

Corollary 4. The variance of the internal profile $I_{n, k}$ tends to infinity if and only if there exists a positive sequence $\omega_{n}$ tending to infinity with $n$ such that

$$
k_{s}+\frac{\omega_{n}}{\log n} \leqslant k \leqslant k_{h}-1-\frac{\omega_{n}}{\log n},
$$

where $k_{s}$ and $k_{h}$ are defined in (13).

Furthermore, the expectation of the internal profile $I_{n, k}$ tends to infinity if and only if there exists a positive sequence $\omega_{n}$ tending to infinity with $n$ such that

$$
\omega_{n} \leqslant k \leqslant k_{h}-1-\frac{\omega_{n}}{\log n} .
$$


Note that the only difference from the central range (14) for the external profile is the additional shift of -1 in the upper bound, a property implied from the fundamental relation

$$
2 I_{n, k}=I_{n, k+1}+B_{n, k+1} .
$$

Since $\operatorname{Var}\left(I_{n, k}\right)=o\left(\left(\mathbb{E}\left(I_{n, k}\right)\right)^{2}\right)$ in the range (58), we thus obtain the following concentration result.

Corollary 5. If $\mathbb{E}\left(I_{n, k}\right) \rightarrow \infty$, then the internal profile is asymptotically concentrated around its expected value:

$$
\frac{I_{n, k}}{\mathbb{E}\left(I_{n, k}\right)} \stackrel{P}{\longrightarrow} 1 \text {. }
$$

Finally, we also expect a central limit theorem in the range (57) where $\operatorname{Var}\left(I_{n, k}\right) \rightarrow \infty$ (similarly to the external profile); this will be addressed in a companion paper.

\section{Applications}

In this section, we apply our results of the profiles to establish the asymptotic two-point concentration of the height and the saturation level in random DSTs, respectively.

\subsection{Height}

We first prove Theorem 3 for the height of random DSTs.

Lemma 10. For all $k$,

$$
1-\sum_{\ell \geqslant 1} 2^{-\ell} \mu_{n, k+\ell} \leqslant \mathbb{P}\left(H_{n} \leqslant k\right)
$$

and for all $k$ with $\mu_{n, k+1} \rightarrow \infty$,

$$
\mathbb{P}\left(H_{n} \leqslant k\right)=\mathcal{O}\left(\frac{1}{\mu_{n, k+1}}\right),
$$

Proof. The proof relies on the first and the second moment method. Noting that $I_{n, k}>0$ if and only if $H_{n}>k$, we have, by the first moment method,

$$
\mathbb{P}\left(H_{n}>k\right)=\mathbb{P}\left(I_{n, k}>0\right) \leqslant \mathbb{E}\left(I_{n, k}\right) .
$$

On the other hand, in view of the relation $I_{n, k}=\sum_{\ell \geqslant 1} 2^{-\ell} B_{n, k+\ell}$, (59) follows from the inequality

$$
\mathbb{P}\left(H_{n}>k\right) \leqslant \sum_{\ell \geqslant 1} 2^{-\ell} \mu_{n, k+\ell} .
$$

For the upper bound (60), since $B_{n, k+1}>0$ implies that $H_{n}>k$, we see that

$$
\mathbb{P}\left(H_{n}>k\right) \geqslant \mathbb{P}\left(B_{n, k+1}>0\right) .
$$

By the second moment method,

$$
\mathbb{P}\left(H_{n} \leqslant k\right) \leqslant \mathbb{P}\left(B_{n, k+1}=0\right) \leqslant \frac{\sigma_{n, k+1}^{2}}{\mu_{n, k+1}^{2}} .
$$

Now, from Theorem 1 and Theorem 2 and the asymptotic growth of $G(z)$ and $H(z)$ from Section 2.3 and Section 3.3, respectively, we deduce that the variance of $B_{n, k}$ is asymptotically of the same order as the mean:

$$
\sigma_{n, k}^{2}=\Theta\left(\mu_{n, k}\right)
$$

for $k$ with $\mu_{n, k} \rightarrow \infty$; compare with Corollary 1. Then (60) follows from (61). 
Theorem 3 is then a consequence of the following two limit results.

Lemma 11. The height of random DSTs satisfies

$$
\lim _{n \rightarrow \infty} \mathbb{P}\left(H_{n} \leqslant k_{H}+1\right)=1 \quad \text { and } \quad \lim _{n \rightarrow \infty} \mathbb{P}\left(H_{n} \leqslant k_{H}-1\right)=0 .
$$

Proof. We first consider the expected value of the external profile around the level $k_{H}$ (defined in (16)) and write

$$
k_{\ell}:=k_{H}+\ell=\left\lfloor\log _{2} n+\sqrt{2 \log _{2} n}-\frac{1}{2} \log _{2} \log _{2} n+\frac{1}{\log 2}\right\rfloor+\ell \quad(\ell \in \mathbb{Z}) .
$$

(Throughout the proof, $\ell$ is fixed unless explicitly stated otherwise.) To prove the asymptotic concentration of the height at $k_{H}$ and $k_{H}+1$, we also need a finer approximation of the mean. By using more terms in the identity of Lemma 2 and (29), we obtain

$$
\mu_{n, k}=2^{k} F\left(2^{-k} n\right)+F^{\prime}\left(2^{-k} n\right)+\mathcal{O}\left(2^{-k} n+n^{-1}\right) .
$$

Since $2^{-k_{\ell}} n \rightarrow 0$, we apply Proposition 1 for the asymptotics of $F\left(2^{-k_{\ell}} n\right)$ (and its derivatives). Note that the saddle-point equation in Proposition 1 has the form $\frac{\rho}{\log \rho}=\frac{2^{k_{\ell}}}{n \log 2}$, or, with $\lambda:=\log _{2} \rho$,

$$
\lambda-\log _{2} \lambda=\sqrt{2 \log _{2} n}-\frac{1}{2} \log _{2} \log _{2} n+\frac{1}{\log 2}+\ell-\theta,
$$

where $\theta=\theta_{n}$ denotes the fractional part of $\log _{2} n+\sqrt{2 \log _{2} n}-\frac{1}{2} \log _{2} \log _{2} n+\frac{1}{\log 2}$. Now, by a direct bootstrapping argument, we obtain

$$
\lambda=\sqrt{2 \log _{2} n}+\frac{1}{\log 2}+\ell+\frac{1}{2}-\theta+\frac{2 \ell+1+\frac{2}{\log 2}-2 \theta}{2 \sqrt{2(\log 2) \log n}}+\mathcal{O}\left(\frac{1+\ell^{2}}{\log n}\right),
$$

which also holds uniformly for $\ell=o\left((\log n)^{1 / 4}\right)$. Substituting this into the asymptotic expansion of $F\left(2^{-k_{\ell}} n\right)$ in Proposition 1, we have

$$
\begin{aligned}
k_{\ell} \log 2+\log F\left(\frac{n}{2^{k_{\ell}}}\right)=- & \sqrt{2 \log _{2} n}(\ell-1-\theta) \log 2-\frac{3 \log \log _{2} n}{4} \\
& +p(\ell-\theta)-P(\lambda)+\mathcal{O}\left(\frac{1+\ell^{2}}{\sqrt{\log n}}\right),
\end{aligned}
$$

again the $O$-term holding uniformly for $\ell=o\left((\log n)^{1 / 4}\right)$, where $P$ is given in (7) and $p(x)$ is the quadratic polynomial:

$$
p(x)=-\frac{\log 2}{2} x^{2}-(1-\log 2) x+1-\frac{1}{2 \log 2}-\frac{5}{8} \log 2-\frac{1}{2} \log \pi .
$$

On the other hand, from (62) and the estimates (by Proposition 1)

$$
\frac{F^{\prime}\left(2^{-k_{\ell}} n\right)}{F\left(2^{-k_{\ell}} n\right)}=\mathcal{O}(\rho)=\mathcal{O}\left(2^{\lambda}\right)
$$

we have

$$
\mu_{n, k_{\ell}}=2^{k_{\ell}} F\left(2^{-k_{\ell}} n\right)\left(1+\mathcal{O}\left(n^{-1} \sqrt{\log n}\right)\right)+o(1) .
$$


Consequently, if $\ell \leqslant 0$, then

$$
\mu_{n, k_{\ell}}=\Omega\left(\frac{2^{\sqrt{2 \log _{2} n}(1+\theta)}}{\left(\log _{2} n\right)^{3 / 4}}\right)+o(1) \rightarrow \infty,
$$

and if $\ell \geqslant 2$, then

$$
\mu_{n, k_{\ell}}=\mathcal{O}\left(\frac{2^{-\sqrt{2 \log _{2} n}(1-\theta)}}{\left(\log _{2} n\right)^{3 / 4}}\right)+o(1) \rightarrow 0 .
$$

Now, by Lemma 10, we show that

$$
\sum_{\ell \geqslant 1} 2^{-\ell} \mu_{n, k_{\ell+1}} \rightarrow 0, \quad \text { and } \quad \mu_{n, k_{0}} \rightarrow \infty,
$$

which will then prove the lemma. The latter follows directly from (65). For the former, we use (64), together with the expansion (63), for $2 \leqslant \ell \leqslant(\log n)^{1 / 5}$, and then the boundedness of $\mu_{n, k_{\ell}}$ (see Corollary 2) for larger $\ell$. This proves (67) and the lemma.

Remark 5. Observe that the only missing case in (65) and (66) is $\ell=1$ for which we have

$$
2^{k_{1}} F\left(2^{-k_{1}} n\right)=\frac{e^{\theta \sqrt{2 \log _{2} n} \log 2+\mathcal{O}(1)}}{\left(\log _{2} n\right)^{3 / 4}} .
$$

Thus, in this case, we have

$$
\mu_{n, k_{1}} \begin{cases}\rightarrow \infty, & \text { if } \theta \geqslant \frac{3 \log _{2} \log _{2} n}{4 \sqrt{2 \log _{2} n}}\left(1+\frac{\omega_{n}}{\log _{2} \log _{2} n}\right), \\ \hdashline 1, & \text { if } \theta=\frac{3 \log _{2} \log _{2} n}{4 \sqrt{2 \log _{2} n}}\left(1 \pm \frac{\mathcal{O}(1)}{\log _{2} \log _{2} n}\right), \\ \rightarrow 0, & \text { if } \theta \leqslant \frac{3 \log _{2} \log _{2} n}{4 \sqrt{2 \log _{2} n}}\left(1-\frac{\omega_{n}}{\log _{2} \log _{2} n}\right)\end{cases}
$$

where $\omega_{n}$ is any sequence tending to infinity.

We see that in almost all cases $\mu_{n, k_{1}} \rightarrow \infty$ and $\mu_{n, k_{1}+1} \rightarrow 0$, meaning that the height is in almost all cases asymptotically equal to $k_{H}+1$; see also [19] where this was observed.

\subsection{Saturation Level}

Recall that the saturation level $S_{n}$ of a binary tree with $n$ internal nodes is defined as the maximal level with $I_{n, k}=2^{k}$, that is, up to level $S_{n}$ the binary tree is complete.

Define $k_{S}$ as

$$
k_{S}=\left\lceil\log _{2} n-\log _{2} \log n\right\rceil,
$$

which is at the lower boundary of the central range (14).

Theorem 6. The distribution of $S_{n}$ is asymptotically concentrated on the two points $k_{S}-1$ and $k_{S}$ :

$$
\mathbb{P}\left(S_{n}=k_{S}-1 \text { or } S_{n}=k_{S}\right) \rightarrow 1, \quad(n \rightarrow \infty) .
$$

Remark 6. Similar to the height, the saturation level $S_{n}$ is concentrated at $k_{S}-1$ in almost all cases.

The proof of Theorem 6 is very similar to that of Theorem 3. The basic observation is that $S_{n}<k$ if and only if $\sum_{\ell \leqslant k} B_{n, \ell}>0$. In particular, if $B_{n, k}>0$ then $S_{n}<k$. Hence, as above, a direct application of the first and the second moment method implies that

$$
1-\sum_{\ell \leqslant k} \mu_{n, \ell} \leqslant \mathbb{P}\left(S_{n} \geqslant k\right) \leqslant \frac{\sigma_{n, k}^{2}}{\mu_{n, k}^{2}} .
$$


By similar arguments as above, Theorem 6 then follows from the limit results:

$$
\lim _{n \rightarrow \infty} \mathbb{P}\left(S_{n} \geqslant k_{S}-1\right)=1 \text { and } \lim _{n \rightarrow \infty} \mathbb{P}\left(S_{n} \geqslant k_{S}+1\right)=0 .
$$

The only difference is that we now use the asymptotic expansion, for $2^{-k} n \rightarrow \infty$,

$$
\mu_{n, k} \sim \sigma_{n, k}^{2} \sim \frac{2^{k}}{Q_{k}}\left(1-2^{-k}\right)^{n} .
$$

\section{Acknowledgment}

We thank the anonymous referees for a careful reading of the paper and for helpful comments and suggestions; one of them offered many critical, well-judged comments and suggestions to the extent of being almost a coauthor of this paper.

\section{References}

[1] D. Aldous and P. Shields (1988). A diffusion limit for a class of random-growing binary trees, Probab. Theory Related Fields, 79, 509-542.

[2] C. W. Anderson (1970). Extreme value theory for a class of discrete distributions, with applications to some stochastic processes, J. Appl. Probab., 7, 99-113.

[3] C. W. Anderson, S. G. Coles and J. Hüsler (1997). Maxima of Poisson-like variables and related triangular arrays, Ann. Appl. Probab., 7, 953-971.

[4] G. E. Andrews (1998). The Theory of Partitions, Cambridge University Press.

[5] E. G. Coffman, Jr. and J. Eve (1970). File structures using hashing functions, Commun. ACM, 13, 427-432.

[6] L. Devroye (1998). Universal limit laws for depths in random trees, SIAM J. Comput., 28, 409-432.

[7] L. Devroye (2005). Universal asymptotics for random tries and PATRICIA trees, Algorithmica, 42, $11-29$.

[8] M. Drmota (2009). Random Trees: An Interplay between Combinatorics and Probability, SpringerWienNewYork, Vienna.

[9] M. Drmota and W. Szpankowski (2011). The expected profile of digital search trees, J. Combin. Theory Ser. A, 118, 1939-1965.

[10] M. Drmota, A. Magner and W. Szpankowski (2018). Asymmetric Rényi problem, Combin. Probab. Comput., accepted for publication.

[11] P. Flajolet (1983), On the performance evaluation of extendible hashing and trie searching, Acta Info. 20, 345-369.

[12] P. Flajolet, X. Gourdon, and P. Dumas (1995). Mellin transforms and asymptotics: harmonic sums. Theoret. Comput. Sci., 144, 3-58.

[13] P. Flajolet and R. Sedgewick (2009). Analytic Combinatorics, Cambridge University Press. 
[14] M. Fuchs, H.-K. Hwang and V. Zacharovas (2014). An analytic approach to the asymptotic variance of trie statistics and related structures, Theor. Comput. Sci., 527, 1-36.

[15] H.-K. Hwang, M. Fuchs and V. Zacharovas (2010). Asymptotic variance of random symmetric digital search trees, Discrete Math. Theor. Comput. Sci. (Special issue in honor of Philippe Flajolet), 12, 103-166.

[16] P. Jacquet and W. Szpankowski (1998). Analytical de-Poissonization and its applications, Theoret. Comput. Sci., 201, 1-62.

[17] P. Jacquet and W. Szpankowski (2015). Analytic Pattern Matching: From DNA to Twitter, Cambridge University Press, Cambridge.

[18] R. Kazemi and M. Vahidi-Asl (2011). The variance of the profile in digital search trees, Discrete Math. Theor. Comput. Sci., 13, 21-38.

[19] C. Knessl and W. Szpankowski (2000). Asymptotic behavior of the height in a digital search tree and the longest phrase of the Lempel-Ziv scheme, SIAM J. Comput., 30, 923-964.

[20] C. Knessl and W. Szpankowski (2002), Limit laws for the height in PATRICIA tries, J. Algorithms 44, 63-97.

[21] C. Knessl and W. Szpankowski (2009). On the average profile of symmetric digital search trees, Online J. Anal. Comb., 4, 14 pp.

[22] D. E. Knuth (1998). The Art of Computer Programming, Volume 3: Sorting and Searching, Second Ed., Addison-Wesley, Reading, MA.

[23] A. G. Konheim and D. J. Newman (1973). A note on growing binary trees, Discrete Math., 4, $57-63$.

[24] G. Louchard (1987). Exact and asymptotic distributions in digital and binary search trees, RAIRO Inform. Thér. Appl., 21, 479-495.

[25] G. Louchard, W. Szpankowski and J. Tang (1999). Average profile of the generalized digital search trees and the generalized Lempel-Ziv Algorithm, SIAM J. Comp., 28, 935-954.

[26] A. Magner and W. Szpankowski (2017). Profile of PATRICIA tries, Algorithmica, 80 (2018), 331397.

[27] A. Magner, C. Knessl and W. Szpankowski (2014). Expected external profile of PATRICIA tries, Proceedings of the Eleventh Workshop on Analytic Algorithmics and Combinatorics (ANALCO), $16-24$.

[28] H. M. Mahmoud. Evolution of Random Search Trees, John Wiley \& Sons Inc., New York, 1992.

[29] R. Neininger and L. Rüschendorf (2004). A general limit theorem for recursive algorithms and combinatorial structures. Ann. Appl. Probab. 14, 378-418.

[30] G. Park, H.-K. Hwang, P. Nicodème and W. Szpankowski (2009). Profile of tries, SIAM J. Comput., 38, 1821-1880.

[31] H. Prodinger (1995). Digital search trees and basic hypergeometric functions, Bulletin of the EATCS, 56, 112-115.

[32] W. Szpankowski (2001). Average Case Analysis of Algorithms on Sequences, Wiley, New York. 


\section{Appendices}

\section{A Appendix A: Proof of Proposition 1}

We give a detailed proof of Proposition 1, which for convenience is re-stated here.

Proposition 1. For each integer $m \geqslant 0$, the $m$ th derivative of $F$ satisfies

$$
F^{(m)}(z)=\frac{\rho^{m+\frac{1}{2}+\frac{1}{\log 2}}}{\sqrt{2 \pi \log _{2} \rho}} \exp \left(-\frac{(\log \rho)^{2}}{2 \log 2}-P\left(\log _{2} \rho\right)\right)\left(1+\mathcal{O}\left(|\log \rho|^{-1}\right)\right),
$$

as $|z| \rightarrow 0$ in the sector $|\arg (z)| \leqslant \varepsilon$, where $P(u)$ is given in (7) and $\rho$ solves the equation

$$
\frac{\rho}{\log \rho}=\frac{1}{z \log 2}
$$

satisfying $|\rho| \rightarrow \infty$ as $|z| \rightarrow 0$.

Proof. Recall that

$$
Q(s):=\prod_{j \geqslant 1}\left(1-2^{-j} s\right) \quad \text { and } \quad Q_{n}:=\prod_{1 \leqslant j \leqslant n}\left(1-2^{-j}\right)=\frac{Q(1)}{Q\left(2^{-n}\right)} .
$$

Also

$$
F(z):=\sum_{j \geqslant 0} \frac{(-1)^{j} 2^{-\left(\begin{array}{c}
j \\
2
\end{array}\right)}}{Q_{j} Q_{\infty}} e^{-2^{j} z} .
$$

Since the Laplace transform $\mathscr{L}[F(z) ; s]$ of $F$ is given by

$$
\mathscr{L}[F(z) ; s]=\prod_{j \geqslant 0} \frac{1}{1+2^{-j} s}=\frac{1}{Q(-2 s)} \quad(\Re(s)>-1),
$$

we have the Laplace inversion formula

$$
F(z)=\frac{1}{2 \pi i} \int_{1-i \infty}^{1+i \infty} \frac{e^{z s}}{Q(-2 s)} \mathrm{d} s
$$

which is valid for $z=x$ where $x>0$ is real. We are interested in the asymptotics of $F(x)$ as $x \rightarrow$ 0 , which is reflected by the large- $|s|$ asymptotics of $\mathscr{L}[F(z) ; s]$. Our approach relies on the Mellin transform techniques and the saddle-point method; see the survey paper [12] for more background tools and applications on Mellin transform. In particular, taking logarithm on both sides of (69) (assuming that $1+2^{-j} s \neq 0$ ), we begin with the Mellin integral representation

$$
\log Q(-2 s)=\sum_{j \geqslant 0} \log \left(1+2^{-j} s\right)=\frac{1}{2 \pi i} \int_{-\frac{1}{2}-i \infty}^{-\frac{1}{2}+i \infty} \frac{\pi s^{-w}}{\left(1-2^{w}\right) w \sin \pi w} \mathrm{~d} w,
$$

because the Mellin transform of $\log (1+s)$ equals

$$
\int_{0}^{\infty} s^{w-1} \log (1+s) \mathrm{d} s=\frac{\pi}{w \sin \pi w}, \quad(\Re(w) \in(-1,0)) .
$$

Note that if $w=u+i v$ and $s=|s| e^{i b}$ with $u, v, b$ real and $|b| \leqslant \pi-\varepsilon$, then

$$
\left|\frac{\pi s^{-w}}{\left(1-2^{w}\right) w \sin \pi w}\right|=\mathcal{O}\left(\frac{|s|^{-u} e^{-|v|(\pi-|b|)}}{\left|1-2^{w}\right||w|}\right),
$$


provided that $|w|$ stays away from the zeros of the denominator. Thus by standard arguments, we deduce that (with $\beta:=\frac{1}{2 \log 2}$ )

$$
\log Q(-2 s)=\beta(\log s)^{2}+\frac{\log s}{2}+P\left(\log _{2} s\right)+J(s),
$$

when $|\arg (s)| \leqslant \pi-\varepsilon$, where the periodic function $P(u)$ has the Fourier series representation

$$
P(u)=\frac{\log 2}{12}+\frac{\pi^{2}}{6 \log 2}-\sum_{j \geqslant 1} \frac{\cos (2 j \pi u)}{j \sinh \frac{2 j \pi^{2}}{\log 2}},
$$

which also defines an analytic function as long as $|\Im(u)|<\pi /(\log 2)$; see Figure 4. Here the remainder $J(s)$ satisfies

$$
\begin{aligned}
J(s) & =\frac{1}{2 \pi i} \int_{\frac{1}{2}-i \infty}^{\frac{1}{2}+i \infty} \frac{\pi s^{-w}}{\left(1-2^{w}\right) w \sin \pi w} \mathrm{~d} w \\
& =\frac{1}{2 \pi i} \int_{-\frac{1}{2}-i \infty}^{-\frac{1}{2}+i \infty} \frac{\pi s^{w}}{\left(1-2^{-w}\right) w \sin \pi w} \mathrm{~d} w \\
& =-\frac{1}{2 \pi i} \int_{-\frac{1}{2}-i \infty}^{-\frac{1}{2}+i \infty} \frac{\pi(2 s)^{w}}{\left(1-2^{w}\right) w \sin \pi w} \mathrm{~d} w \\
& =-\log Q\left(-\frac{1}{s}\right) .
\end{aligned}
$$

We thus have the identity:
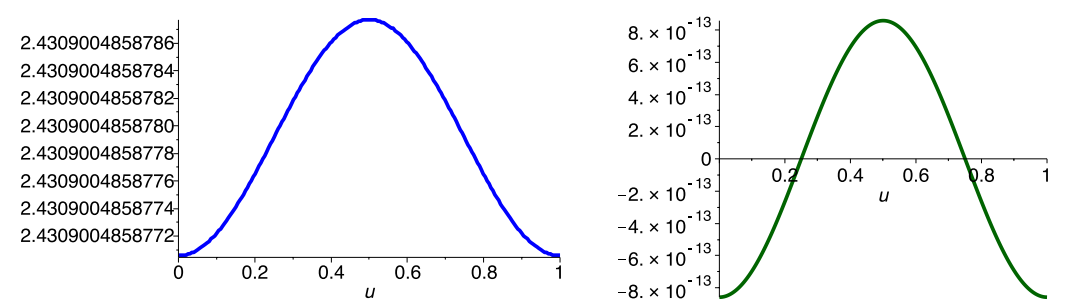

Figure 4: $P(u)$ in the unit interval (left) and the fluctuating part of $P(u)$ (right).

$$
Q(-2 s)=\frac{\sqrt{s} e^{\beta(\log s)^{2}+P\left(\log _{2} s\right)}}{Q\left(-\frac{1}{s}\right)}
$$

or

$$
\prod_{j \geqslant 0}\left(1+\frac{s}{2^{j}}\right)=\sqrt{s} e^{\beta(\log s)^{2}+P\left(\log _{2} s\right)} \prod_{j \geqslant 1} \frac{1}{1+\frac{1}{2^{j} s}},
$$

which indeed holds, by analytic continuation, as long as $s \in \mathbb{C} \backslash(-\infty, 0]$. In particular, for large $|s|$ with $|\arg (s)| \leqslant \pi-\varepsilon$,

$$
J(s)=-\frac{1}{s}+\frac{1}{6 s^{2}}-\frac{1}{21 s^{3}}+\frac{1}{60 s^{4}}+\mathcal{O}\left(|s|^{-5}\right) .
$$

It follows, by substituting the asymptotic approximation (34), that

$$
F(x)=\frac{1}{2 \pi i} \int_{1-i \infty}^{1+i \infty} s^{-\frac{1}{2}} e^{x s-\beta(\log s)^{2}-P\left(\log _{2} s\right)}\left(1+\mathcal{O}\left(|s|^{-1}\right)\right) \mathrm{d} s .
$$


Now, the asymptotics of $F(x)$ as $x \rightarrow 0$ is obtained by a standard application of the saddle-point method. Therefore, we move the line of integration to $\Re(s)=\rho$, where $\rho>0$ solves the saddle-point equation $\frac{\log \rho}{\rho}=\frac{x}{2 \beta}$. Note that this does not change the value of the integral which is either clear from the domain of the Laplace transform of $f(z)$ or can also be seen directly since the integrand over the horizontal line segments of distance $T \gg 1$ from the positive real axis (and contained in a cone with $|\arg (s)| \leqslant \pi-\varepsilon)$ is bounded above by

$$
T^{-\frac{1}{2}} e^{x \Re(s)-\beta(\log T)^{2}},
$$

implying that the integral along such lines is of order

$$
T^{-\frac{1}{2}} \exp \left(-\beta(\log T)^{2}\right),
$$

which decays to 0 as $T$ tends to infinity. Thus, (with $s \mapsto \rho(1+i t)$ )

$$
F(x)=\frac{\rho^{\frac{1}{2}} e^{\rho x}}{2 \pi} \int_{-\infty}^{\infty} \frac{e^{i \rho t x-\beta(\log (\rho(1+i t)))^{2}-P\left(\log _{2}(\rho(1+i t))\right)}}{\sqrt{1+i t}}\left(1+\mathcal{O}\left(\frac{1}{\rho|1+i t|}\right)\right) \mathrm{d} t .
$$

By a direct iterative argument, we obtain, with $R:=\frac{2 \beta}{x}$,

$$
\rho=R\left(\log R+\log \log R+\frac{\log \log R}{\log R}-\frac{(\log \log R)^{2}-2 \log \log R}{2(\log R)^{2}}+\mathcal{O}\left(\frac{|\log \log R|^{3}}{|\log R|^{3}}\right)\right) .
$$

Then we split the integral into two parts:

$$
F(x)=\frac{\rho^{\frac{1}{2}} e^{\rho x}}{2 \pi}\left(\int_{|t| \leqslant t_{0}}+\int_{|t|>t_{0}}\right) \frac{e^{i \rho t x-\beta(\log (\rho(1+i t)))^{2}-P\left(\log _{2}(\rho(1+i t))\right)}}{\sqrt{1+i t}}\left(1+\mathcal{O}\left(\frac{1}{\rho|1+i t|}\right)\right) \mathrm{d} t,
$$

where $t_{0}=(\log \rho)^{-\frac{2}{5}}$. Since

$$
\Re\left((\log (\rho(1+i t)))^{2}\right)=(\log \rho)^{2}+(\log \rho) \log \left(1+t^{2}\right)+\frac{1}{4}\left(\log \left(1+t^{2}\right)\right)^{2}-\arctan (t)^{2}
$$

is a monotonic function of $|t|$ for fixed large $\rho$, we have

$$
\begin{aligned}
\int_{|t|>t_{0}} & \frac{e^{i \rho t x-\beta(\log (\rho(1+i t)))^{2}-P\left(\log _{2}(\rho(1+i t))\right)}}{\sqrt{1+i t}} \mathrm{~d} t \\
= & \mathcal{O}\left(e^{-\beta(\log \rho)^{2}} \int_{\log \left(1+t_{0}^{2}\right)}^{\infty} w^{-\frac{1}{2}} e^{-\beta\left(w \log \rho+\frac{1}{4} w^{2}\right)+w} \mathrm{~d} w\right) \\
= & \mathcal{O}\left(e^{-\beta(\log \rho)^{2}-\varepsilon(\log \rho)^{\frac{1}{5}}}\right),
\end{aligned}
$$

for some $\varepsilon>0$. Now by the local expansions

$$
\begin{aligned}
i \rho t x & -\beta(\log (\rho(1+i t)))^{2} \\
& =-\beta(\log \rho)^{2}-\beta(\log \rho-1) t^{2}+\frac{\beta}{3}(2 \log \rho-3) i t^{3}+\mathcal{O}\left(t^{4} \log \rho\right),
\end{aligned}
$$

and

$$
e^{-P\left(\log _{2}(\rho(1+i t))\right)}=e^{-P\left(\log _{2} \rho\right)}\left(1-\frac{P^{\prime}\left(\log _{2} \rho\right)}{\log 2} i t+\mathcal{O}\left(|t|^{2}\right)\right),
$$


for $|t| \leqslant t_{0}$, we deduce that the integral with $|t| \leqslant t_{0}$ is asymptotic to

$$
\begin{aligned}
F(x) & =\frac{\rho^{\frac{1}{2}} e^{\rho x}}{2 \pi} \int_{|t| \leqslant t_{0}} \frac{e^{i \rho t x-\beta(\log (\rho(1+i t)))^{2}-P\left(\log _{2}(\rho(1+i t))\right)}}{\sqrt{1+i t}}\left(1+\mathcal{O}\left(\frac{1}{\rho|1+i t|}\right)\right) \mathrm{d} t \\
& =\frac{\rho^{\frac{1}{2}} e^{\rho x-\beta(\log \rho)^{2}-P\left(\log _{2} \rho\right)}}{2 \sqrt{\pi \beta \log \rho}}\left(1+\mathcal{O}\left((\log \rho)^{-1}\right)\right) .
\end{aligned}
$$

Similarly, we also have

$$
F^{(m)}(x)=\frac{\rho^{m+\frac{1}{2}} e^{\rho x-\beta(\log \rho)^{2}-P\left(\log _{2} \rho\right)}}{2 \sqrt{\pi \beta \log \rho}}\left(1+\mathcal{O}\left(m^{2}(\log \rho)^{-1}\right)\right),
$$

uniformly as $x \rightarrow 0$ and $m=o(\sqrt{\log \rho})$.

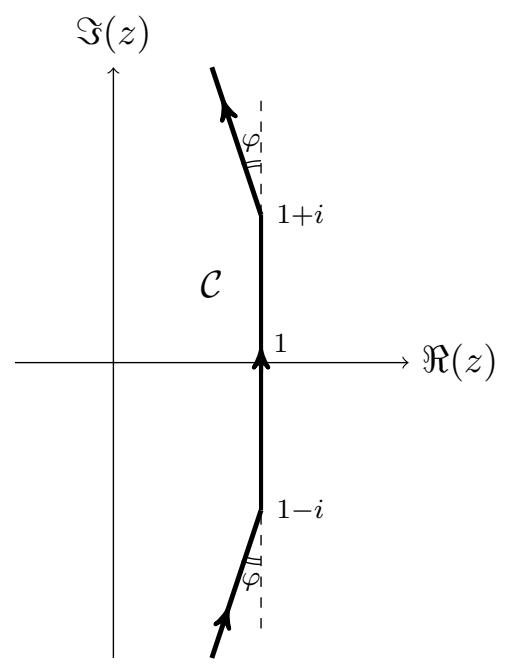

Figure 5: The contour of integration in the integral representation of $F(z)$ when $z$ is complex.

We now look at the situation when $z=x e^{i \theta}$ with $\theta \neq 0$ and $|\theta| \leqslant \varepsilon$. Here, (70) is no longer valid since the integral diverges. However, by the same idea of the Hankel contour used for extending the Gamma function, we can deform the original integration line into the following one:

$$
\begin{aligned}
\mathcal{C}:=\left\{z=1-i+e^{-i\left(\frac{\pi}{2}+\varphi\right)} u: u \geqslant 0\right\} & \\
& \cup\{z=1+i u:-1<u<1\} \cup\left\{z=1+i+e^{i\left(\frac{\pi}{2}+\varphi\right)} u: u \geqslant 0\right\},
\end{aligned}
$$

where $\varepsilon<\varphi$; see Figure 5. Then, we use (34) and make the substitution

$$
\begin{aligned}
F(z) & =\frac{1}{2 \pi i} \int_{\mathcal{C}} s^{-\frac{1}{2}} e^{x e^{i \theta} s-\beta(\log s)^{2}-P\left(\log _{2} s\right)}\left(1+\mathcal{O}\left(|s|^{-1}\right)\right) \mathrm{d} s \\
& =\frac{e^{-\frac{1}{2} i \theta}}{2 \pi i} \int_{e^{i \theta} \mathcal{C}} s^{-\frac{1}{2}} e^{x s-\beta(\log s-i \theta)^{2}-P\left(\log _{2}\left(s e^{-i \theta}\right)\right)}\left(1+\mathcal{O}\left(|s|^{-1}\right)\right) \mathrm{d} s
\end{aligned}
$$

where $e^{i \theta} \mathcal{C}$ denotes the image of $\mathcal{C}$ under the mapping $s \mapsto e^{i \theta} s$. Note that the solution to the saddlepoint equation

$$
\frac{\log \rho(z)}{\rho(z)}=\frac{z}{2 \beta}=\frac{e^{i \theta}}{x}
$$


where $R:=\frac{2 \beta}{x}$, satisfies asymptotically for small $x$

$$
\rho(z)=R e^{-i \theta}\left(\log R+\log \log R-i \theta+\frac{\log \log R-i \theta}{\log R}+\mathcal{O}\left(\frac{|\log \log R|^{2}}{|\log R|^{2}}\right)\right) .
$$

In particular $(\rho=\rho(|z|))$,

$$
\rho(z)=\rho e^{-i \theta}\left(1-\frac{i \theta}{\log R}+\frac{(\log \log R-1) i \theta}{(\log R)^{2}}+\mathcal{O}\left(\frac{|\log \log R|^{2}}{|\log R|^{3}}\right)\right) .
$$

Since $|\theta| \leqslant \varepsilon$, we now deform the integration contour again into the vertical line $\Re(s)=\rho$ (which again does not change the value of the integral as can be seen by a similar argument as above) and proceed as before:

$$
\begin{aligned}
F(z)= & \frac{e^{-\frac{1}{2} i \theta}}{2 \pi i}\left(\int_{\begin{array}{c}
s=\rho \cdot(1+i t) \\
|t| \leqslant t_{0}
\end{array}}+\int_{\begin{array}{c}
s=\rho \cdot(1+i t) \\
|t|>t_{0}
\end{array}}\right) \\
& s^{-\frac{1}{2}} e^{x s-\beta(\log s-i \theta)^{2}-P\left(\log _{2} s-i \theta /(\log 2)\right)}\left(1+\mathcal{O}\left(|s|^{-1}\right)\right) \mathrm{d} s .
\end{aligned}
$$

By the local expansion

$$
\begin{aligned}
x \rho i t-\beta(\log (\rho \cdot(1+i t))-i \theta)^{2}= & -\beta(\log \rho-i \theta)^{2}-2 \beta \theta t-\beta(\log \rho-1-i \theta) t^{2} \\
& +\frac{\beta}{3}(2 \log \rho-3-2 i \theta) i t^{3}+\mathcal{O}\left((\log \rho) t^{4}\right),
\end{aligned}
$$

and the relations $(a \in \mathbb{R}, b>0)$

$$
\frac{1}{2 \pi} \int_{-\infty}^{\infty} t^{m} e^{-a t-b t^{2}} \mathrm{~d} t=\frac{(-1)^{m} e^{\frac{a^{2}}{4 b}}}{2^{m+1} \sqrt{\pi b}} \sum_{0 \leqslant \ell \leqslant\left\lfloor\frac{1}{2} m\right\rfloor} \frac{m ! a^{m-2 \ell}}{\ell !(m-2 \ell) ! b^{m-\ell}} \quad(m=0,1, \ldots),
$$

we deduce that the first integral on the right-hand side of (74) is asymptotic to

$$
\begin{gathered}
\frac{\rho^{\frac{1}{2}} e^{-\frac{1}{2} i \theta-P\left(\log _{2}\left(\rho e^{-i \theta}\right)\right)+x \rho-\beta(\log \rho-i \theta)^{2}-\frac{\beta^{2} \theta^{2}}{\log \rho-1-i \theta}}}{2 \sqrt{\pi \beta(\log \rho-1-i \theta)}}\left(1+\mathcal{O}\left((\log \rho)^{-1}\right)\right) \\
=\frac{\rho^{\frac{1}{2}} e^{-\frac{1}{2} i \theta-P\left(\log _{2}\left(\rho e^{-i \theta}\right)\right)+x \rho-\beta(\log \rho-i \theta)^{2}}}{2 \sqrt{\pi \beta \log \rho}}\left(1+\mathcal{O}\left((\log \rho)^{-1}\right)\right) .
\end{gathered}
$$

By (73), the right-hand side is asymptotic to

$$
\frac{\rho(z)^{\frac{1}{2}} e^{-P\left(\log _{2} \rho(z)\right)+z \rho(z)-\beta(\log \rho(z))^{2}}}{2 \sqrt{\pi \beta \log \rho(z)}}\left(1+\mathcal{O}\left(|\log \rho(z)|^{-1}\right)\right) .
$$

It remains to prove the smallness of the other integral in (74), which is bounded above by

$$
\begin{aligned}
& \int_{\substack{s=\rho \cdot(1+i t) \\
|t|>t_{0}}} s^{-\frac{1}{2}} e^{x s-\beta(\log s-i \theta)^{2}-P\left(\log _{2}\left(s e^{-i \theta}\right)\right)} \mathrm{d} s \\
& \quad=\mathcal{O}\left(\rho^{\frac{1}{2}} e^{x \rho} \int_{t_{0}}^{\infty}\left(1+t^{2}\right)^{-\frac{1}{4}} e^{-\beta\left(\left(\log \rho+\frac{1}{2} \log \left(1+t^{2}\right)\right)^{2}-(\theta-\arctan (t))^{2}\right)} \mathrm{d} t\right) .
\end{aligned}
$$

The factor $(\theta-\arctan (t))^{2}$ being bounded for $t$ in the range of integration, we obtain

$$
\mathcal{O}\left(\rho^{\frac{1}{2}} e^{x \rho} \int_{t_{0}}^{\infty}\left(1+t^{2}\right)^{-\frac{1}{4}} e^{-\beta\left(\log \rho+\frac{1}{2} \log \left(1+t^{2}\right)\right)^{2}} \mathrm{~d} t\right)=\mathcal{O}\left(\rho^{\frac{1}{2}} e^{x \rho-\beta(\log \rho)^{2}-\varepsilon(\log \rho)^{\frac{1}{5}}}\right),
$$


which, by (73), is majorized by

$$
\mathcal{O}\left(|\rho(z)|^{\frac{1}{2}} e^{\Re\left(z \rho(z)-\beta(\log \rho(z))^{2}\right)-\varepsilon|\log \rho(z)|^{\frac{1}{5}}}\right) .
$$

We thus obtain the approximation

$$
F(z)=\frac{\rho(z)^{\frac{1}{2}} e^{z \rho(z)-\beta(\log \rho(z))^{2}-P\left(\log _{2} \rho(z)\right)}}{2 \sqrt{\pi \beta \log \rho(z)}}\left(1+\mathcal{O}\left(|\log \rho(z)|^{-1}\right)\right),
$$

uniformly as $|z| \rightarrow 0$ in the sector $|\arg (z)| \leqslant \varepsilon$. The proof for the $m$ th derivative of $F(z)$ is similar as above.

\section{B Appendix B: Proof of (47)}

It suffices to prove (47) for $m=0$ because the general statement follows by well-known properties of the Laplace transform.

First, by straightforward bounds, we have $G(x)=\mathcal{O}(1)$ for $x \geqslant 0$, and thus the Laplace transform $\mathscr{L}[G(x) ; s]$ exists for $\Re(s)>0$. Moreover, for the computation of this Laplace transform, we can interchange the integral and the series, and obtain

$$
\mathscr{L}[G(x) ; s]=\sum_{j, r \geqslant 0} \sum_{0 \leqslant h, \ell \leqslant j} \frac{(-1)^{r+h+\ell_{2}-j-\left(\begin{array}{c}
r \\
2
\end{array}\right)-\left(\begin{array}{c}
h \\
2
\end{array}\right)-\left(\begin{array}{c}
\ell \\
2
\end{array}\right)+2 h+2 \ell}}{Q_{\infty} Q_{r} Q_{h} Q_{j-h} Q_{\ell} Q_{j-\ell}} \cdot \frac{1}{\left(s+2^{h}+2^{\ell}\right)^{2}\left(s+2^{j+r}\right)} .
$$

Now, as in the proof of Proposition 1,

$$
\sum_{r \geqslant 0} \frac{(-1)^{r} 2^{-\left(\begin{array}{c}
r \\
2
\end{array}\right)}}{Q_{\infty} Q_{r}\left(s+2^{j+r}\right)}=\frac{1}{2^{j} Q\left(-2^{1-j} s\right)} .
$$

Plugging this into the above expression gives

$$
\mathscr{L}[G(x) ; s]=\sum_{j \geqslant 0} \frac{1}{4^{j} Q\left(-2^{1-j} s\right)} \sum_{0 \leqslant h, \ell \leqslant j} \frac{(-1)^{h+\ell} 2^{-\left(\begin{array}{c}
h \\
2
\end{array}\right)-\left(\begin{array}{l}
\ell \\
2
\end{array}\right)+2 h+2 \ell}}{Q_{h} Q_{j-h} Q_{\ell} Q_{j-\ell}} \cdot \frac{1}{\left(s+2^{h}+2^{\ell}\right)^{2}} .
$$

Next, by differentiating (23) twice,

$$
\tilde{M}_{k, 1}^{\prime \prime}(z)=2^{-k} \sum_{0 \leqslant \ell \leqslant k} \frac{(-1)^{\ell} 2^{-\left(\begin{array}{c}
\ell \\
2
\end{array}\right)+2 \ell}}{Q_{\ell} Q_{k-\ell}} e^{-2^{\ell-k} z}
$$

and thus

$$
\begin{aligned}
\tilde{g}_{j}^{*}(s)=\mathscr{L}\left[z\left(\tilde{M}_{j, 1}^{\prime \prime}(z)\right)^{2} ; s\right] & =4^{-j} \sum_{0 \leqslant h, \ell \leqslant j} \frac{(-1)^{h+\ell} 2^{-\left(\begin{array}{c}
h \\
2
\end{array}\right)-\left(\begin{array}{l}
\ell \\
2
\end{array}\right)+2 h+2 \ell}}{Q_{h} Q_{j-h} Q_{\ell} Q_{j-\ell}} \cdot \frac{1}{\left(s+2^{h-j}+2^{\ell-j}\right)^{2}} \\
& =\sum_{0 \leqslant h, \ell \leqslant j} \frac{(-1)^{h+\ell_{2}} 2^{-\left(\begin{array}{c}
h \\
2
\end{array}\right)-\left(\begin{array}{c}
\ell \\
2
\end{array}\right)+2 h+2 \ell}}{Q_{h} Q_{j-h} Q_{\ell} Q_{j-\ell}} \cdot \frac{1}{\left(2^{j} s+2^{h}+2^{\ell}\right)^{2}} .
\end{aligned}
$$

Finally, substituting this into (75) gives

$$
\mathscr{L}[G(x) ; s]=\sum_{j \geqslant 0} 4^{-j} \frac{\tilde{g}_{j}^{*}\left(2^{-j} s\right)}{Q\left(-2^{1-j} s\right)} \quad(\Re(s)>0),
$$

as claimed. 